\title{
Viscoelastic flow instabilities in static mixers: onset and effect on the mixing efficiency
}

\author{
S. Migliozzi, L. Mazzei, a) and P. Angelia) \\ Chemical Engineering Department, University College London \\ Torrington Place, WCIE 7JE, London, UK
}

(Dated: 21 December 2020)

\begin{abstract}
Elastic instabilities are identified as flow instabilities occurring in the presence of low inertial effects, induced by the combination of strong elastic forces with nonlinearities of the flow. In continuous flow laminar mixing applications, the onset of these instabilities is likely to occur in the window of applied flow rates; therefore, it is important to understand the effects of their onset on the process efficiency. In this work, we investigated experimentally the onset of elastic instabilities in two tubular static mixers with different geometric features, i.e. the Kenics helical mixer and the SMB$\mathrm{R}$ mixer, the latter characterised by a double X-shaped bars geometry. We obtained concentrations maps at various mixer lengths by means of Planar Laser Induced Fluorescence techniques. To deduce a generalised effect of the fluid elasticity on the mixing patterns, we tested three fluids with different rheological behaviour - a Boger fluid and two shear-thinning fluids. For all cases, we observed deviations from the Newtonian benchmark as soon as the Deborah number exceeded unity, even though different transitions occurred as the mean flow rate increased. The effect of the instability on the mixing patterns strongly depended on the different kinematics induced by the two geometries: for the helical mixer the typical lamellar structure is not recovered and the two liquid streams remain unmixed, whilst for the SMB-R the concentration maps are strongly unsteady, showing temporally and spatially chaotic fluctuations of the mass fraction. In both cases, the instabilities worsen the mixing efficiency compared to the Newtonian case.
\end{abstract}

\section{INTRODUCTION}

Mixing operations in laminar flow conditions are encountered in many industrial applications involving complex fluids. In these conditions, a rigorous way to characterize the kinematics of fluid mixing is extremely important, since local segregated areas are more likely to occur than in turbulent flows, where the unsteady velocity field continuously reorients the fluid elements in the Lagrangian reference frame, promoting mixing in the fluid bulk ${ }^{1}$. Interesting results have been derived by applying concepts developed for the study of chaotic dynamical systems to laminar mixing applications ${ }^{2}$. Experimental and computational studies have demonstrated that mixing in laminar conditions can be induced by a periodicity in the flow and that the evolution of partially mixed structures can be described based on the stretching and folding of fluid elements placed into the flow, following the concept of the 'baker's transformation' ${ }^{3-6}$. Stretching directly affects the generation rate of intermaterial area and the distribution of striation thickness in the flow, while folding is central for putting in contact the different liquid streams, thus promoting mixing in a direction orthogonal to the main velocity field.

Static mixers represent the direct technological application of this principle. Identified also as motionless mixers, they consist of a series of fixed elements, placed in a pipe with the intent of re-orientating the flow to promote radial mixing. Significant interest has developed in the use of motionless mixers for laminar flow applications, driven by the necessity to intensify the traditional batch processes by substituting them with continuous processes. Their popularity stems from their

a) The authors to whom correspondence may be addressed: p.angeli@ucl.ac.uk,1.mazzei@ucl.ac.uk. relatively simple implementation (energy is provided through standard pumping equipment) and design flexibility compared to other in-line mixing devices (e.g. extruders); besides, if properly designed, static mixers offer highly reliable performance over a wide range of operating conditions ${ }^{7,8}$. Given the technological relevance of these devices, numerous studies can be found in the literature focusing on the general performance of different designs ${ }^{9-15}$ as well as attempts to find a generalised theoretical approach for the design of the mixers based on the study of chaotic systems. Most of these studies focus on Newtonian or generalised Newtonian fluids ${ }^{16-19}$.

In spite of the ubiquity of complex fluids in industrial applications, investigations on the effect of non-linear rheological behaviour on the mixing performance in continuous flow systems are scarce and primarily focused on inelastic shearthinning fluids ${ }^{10,14,20-22}$. This is due to the relatively simple implementation of generalized Newtonian constitutive equations in CFD codes. The performance of in-line mixing devices has been investigated much less experimentally, because of the high level of sophistication and resources needed to capture the mixing dynamics with the same level of detail as that achieved numerically. But the importance of experimental observations should not be underestimated when dealing with complex fluids. Indeed, a recent experimental work of Lim et al. ${ }^{23}$ highlighted a modest detrimental effect of CMC solutions (widely used as inelastic shear-thinning model fluids) on the mixing rate in a SMX mixer, a finding that is opposite to that of many numerical studies, which usually show no effects or positive effects when inelastic shear thinning fluids are used ${ }^{14,24,25}$.

The influence of complex rheological behaviour such as fluid elasticity, as well as the combination of elasticity and shear-thinning, on mixing dynamics is still not fully understood. The strong nonlinearities induced by the presence of polymer molecules can severely complicate viscoelastic 
flows. Polymer chains confer an extra tensile stress to the fluid streamlines, which depends on the flow history through a characteristic relaxation time $\lambda^{26}$. In the low Reynolds regime, typical of laminar mixing applications, pure elastic instabilities can arise from the interaction of curvilinear streamlines with the extra elastic stresses, which act along the streamlines. Such instabilities have been observed experimentally for simple viscometric ${ }^{27-30}$ and more complex flows ${ }^{31-37}$. Theoretical works based on linear stability analysis of nonlinear rheological constitutive models showed the possibility of predicting the onset of flow bifurcations induced by pure elasticity and their nature for simple flows, i.e. cone-plate and parallelplate rheometric flows and Taylor-Couette flow ${ }^{30,38,39}$. For more complex two-dimensional geometries, McKinley and co-workers ${ }^{40,41}$ introduced a dimensionless criterion to analyse the onset of purely elastic instabilities and their sensitivity to the flow geometry and fluid rheological properties, which in simple flows they proved to reduce to well-established results.

For mixing applications, the presence of flow bifurcations induced by elasticity is a significant issue; however, a direct translation of the theoretical findings cited above is arduous, given the intrinsic complexity of the flow kinematics in traditional mixers. To these days, the effect of elasticity is controversial. Some fundamental insights have been gained by studying viscoelastic flows in 2-D or simplified 3-D geometries. Generally, elastic fluids seem to induce poorer mixing as the shear rate is increased owing to an increased resistance to stretching, which induces the formation of large unmixed regions ${ }^{42,43}$. However, depending on the flow conditions, in some geometries elasticity can improve mixing thanks to the onset of elastic turbulence $e^{44-48}$. These discrepancies underline how the effect of nonlinear rheology on mixing strongly depends on the mixing protocol and is difficult to predict theoretically for complex flows, thus making experimental investigations necessary. To the best of the authors' knowledge, the recent paper of Ramsay et al. ${ }^{49}$ is the only study available in the literature on the effect of elasticity on the mixing performance of static mixers. They found that viscoelasticity significantly affects the mixing patterns at the exit of a 6-element Kenics mixer by inducing large segregated zones and temporal fluctuations of concentration, which they attributed to the presence of elastic instabilities. Following these observations, further experimental investigations are required to clearly establish the onset of the instabilities and their effects on the mixing efficiency.

The aim of this work is to investigate experimentally the onset of elastic instabilities in static mixers and their effect on the topology of the mixing patterns, depending on the specific geometry of the mixer and the fluid rheology. Two different mixer geometries were selected to highlight the impact of different flow fields on the fluid response, which, in turn, influence the mixing dynamics, i.e. the Kenics helical mixer and the SMB-R mixer. Planar Laser Induced Fluorescence (PLIF) measurements were performed to capture instantaneous concentration maps for different operating conditions, whilst pressure drop measurements allowed continuous monitoring of the flow resistance. Three different polymeric solutions were used to investigate the effect of pure elasticity and the combination of elasticity and shear-thinning behaviour. All experimental observations are discussed and summarised using the relevant dimensionless groups.

The article is organised as follows. In Section II, we describe the experimental setup and the techniques implemented, followed by the rheological characterisation of the model fluids employed and the definition of the dimensionless numbers. The experimental results are then presented and discussed in Section III for each of the two geometries. Finally, we report our conclusions in Section IV.

\section{MATERIALS AND METHODS}

\section{A. Experimental apparatus and mixer geometries}

Two geometries were selected for the experimental investigations, the Kenics helical mixer (Euromixers Ltd) and the SMB-R mixer (Bayer Licence, Stamixco Ltd). Both geometries were chosen for direct insertion in a circular pipe. The first mixer is one of the standard geometries used for in-line mixing applications. Owing to its simplicity, this geometry has been extensively studied in the literature $e^{9,11,50-52}$, thus representing a good benchmark for this study. The mixer is constituted by a total of six metal helical elements, with twisting angle of 180 and aspect ratio $A R=L / D=1.35$, with $L$ and $D$ being the length and the diameter of the mixing element, respectively. To ensure correct functioning, elements with opposite twisting direction must be inserted one after the other with the leading edge at 90 with the trailing edge of the previous element. The elements are welded together in couples, hence experimental investigations can be carried out for mixer lengths of two, four or six elements. The other geometry belongs to a different type of inline mixers design, commonly used in polymer processing ${ }^{8}$, characterised by higher compactness and a more complex geometry of its internal surfaces. The mixer has a total of eight detachable elements and, as for the helical mixer, each consecutive element is rotated at 90 relative to the previous. The characteristic dimensions and CAD drawings of both geometries can be found in the supplementary information (SI-1).

Experiments were carried out in a modular continuous flow rig to allow design flexibility. A schematic of the experimental setup is shown in Fig. 1. For each fluid tested, two liquid streams, one dyed and one undyed, were pumped separately, but with equal volumetric flow rates, through two gear pumps (AxFlow Ltd). For each liquid stream, a gear flow meter (Max Machinery, Inc.) allowed continuous monitoring of the flow rates before the streams entered the horizontal test section, which was made of transparent poly-(methyl methacrylate) (PMMA) to allow optical access. The two streams were brought together through a Y-junction to form one stream with a central vertical interface between the dyed and the undyed materials. The junction was designed to maintain constant average velocities in the flow upstream and downstream of the junction (inset in Fig. 1). The horizontal rig (ID 25.4 $\mathrm{mm}$ ) consisted of three modular sections, (i) a mixing section, (ii) a visualization section, and (iii) an outlet section. 
The visualisation section was encased in a PMMA rectangular box, which was filled with a glycerol/water mixture to match the refractive index of PMMA and avoid any optical artefacts induced by the curvature of the pipe, whilst the outlet section was a custom-made L-shaped junction with a vertical flat surface that allowed direct observation of the pipe cross-section. All the sections had the same internal diameter and were assembled together through external flanges to assure perfect alignment and minimise disturbances in the flow. For each mixer geometry, the mixing section was fabricated to assure precise fitting on the static elements. To maintain in place the mixing elements and ensure consistency of their position along the pipe length for different experiments, a small concentric restriction was incorporated at the end of the mixing section (local reduction of the internal diameter $\sim 1$ $\mathrm{mm})$. This implies that the exit of the last mixing element was always fixed, independently of the number of elements inserted. Pressure ports were drilled at the inlet and outlet of the mixing sections allowing pressure drop measurements through a high performance differential pressure transmitter (model 266MST, ABB). All experiments were conducted at a controlled room temperature of $21 \mathrm{C}$. The fluid temperature was continuously monitored through a thermocouple inserted in an additional port placed at the end of the mixing unit, confirming that the operating temperature was maintained at 21 C.

\section{B. Planar Laser Induced Fluorescence (PLIF) setup}

To capture the instantaneous concentration maps induced by the two mixers, planar laser induced fluorescence (PLIF) experiments were carried out at different operating conditions. Rhodamine 6G (R-6G) was added as fluorescent dye in one of the fluid streams at a concentration of $8.5 \times 10^{-8} \mathrm{~mol} / \mathrm{L}$, which is within the range of concentrations for which $I_{G} \propto c$, where $I_{G}$ and $c$ are the greyscale intensity of the image and the dye concentration, respectively (see SI-2 for details on the calibration method). The PLIF system consisted of a continuous laser (Diode-Pumped Solid-State series with output power of $3000 \mathrm{~mW}$ and wavelength $\lambda=532 \mathrm{~nm}$, Laserglow Technologies) and a high speed camera (Phantom v1212, Ametek Inc.) equipped with a mono-zoom Nikon Lens, which gave a spatial resolution of $24 \mu \mathrm{m} / \mathrm{pixel}$, and an orange filter with cut-on wavelength of $570 \mathrm{~nm}$, which eliminates any reflection on the pipe or spurious light of the laser. The laser unit was equipped with two cylindrical lenses $(25 \mathrm{~mm}$ and $15 \mathrm{~mm})$ to create a narrow laser plane of $1 \mathrm{~mm}$ thickness. The generated horizontal plane was reflected on a 45 silver coated mirror to form a vertical plane, perfectly perpendicular to the pipe cross-section (see schematic in Fig. 1). The laser sheet and the camera focal plane were fixed downstream of the mixing elements, at $3 / 4$ of the total length of the visualisation box (approximately $20 \mathrm{~cm}$ from the exit of the last mixing element). This choice is related to the necessity of reducing reflections from the pipe walls by minimising the distance between the camera lens and the focal plane. Preliminary tests, conducted by changing the z-position of the focal plane along the visu- alisation section, assured that the maps remained unchanged after exiting the last mixing element. In addition, at the lowest flow rate investigated, the Peclet number was $O\left(10^{7}\right)$, thus ensuring that any effect of diffusion was negligible in the axial direction after the mixing elements for all experiments conducted.

Images were recorded continuously with a frame rate of 100 fps from the start-up of the pumps. Flow conditions were further monitored through pressure drop measurements (acquisition rate of $5000 \mathrm{~Hz}$ ) to ensure maps were recorded after steady state conditions were reached. To map the onset of the instabilities and understand the changes in the mixing mechanism induced by different rheological properties, the experiments were repeated for each fluid at increasing flow rates, first at the minimum number of elements possible (i.e. 2 for the Kenics and 1 for the SMB-R), and then gradually adding more elements. The images recorded over the entire steadystate period were then averaged, when the concentration profiles did not present any time variation, and normalised to obtain greyscale maps of the mass fraction of dyed stream $C$ using the following relation ${ }^{53}$ :

$$
C(x, y)=\frac{I(x, y)-I_{D}(x, y)}{I_{B}(x, y)-I_{D}(x, y)}
$$

where $I$ is the recorded intensity and $I_{D}$ and $I_{B}$ are the intensity values of the dark and the bright reference images, respectively. For the unsteady cases, the same normalisation was applied to each single frame, without averaging consecutive frames. All the intensity values were normalised using the corresponding pixel value to remove any bias related to inhomogeneities in the illumination plane.

The flow resistance was also evaluated in all conditions tested. A friction factor $f_{F}$ was calculated from the pressure drop measurements to obtain a proper comparison between different fluids, using the following relation ${ }^{54}$ :

$$
f_{F}=\frac{1}{2} \frac{\Delta P}{N_{e}\left(\frac{L}{D}\right) \rho\left(\frac{U}{\varepsilon}\right)^{2}}
$$

where $\Delta P$ is the pressure drop measured over the whole mixer length, $N_{e}$ is the total number of mixing elements, $L$ and $D$ are the length and diameter of the single mixing element, respectively, $\rho$ is the density of the fluid, $U$ is the mean velocity evaluated considering an empty pipe and $\varepsilon$ is the porosity of the mixer.

\section{Fluids preparation}

Four fluids were used for the experimental investigation: one Newtonian fluid as benchmark case, one Boger fluid and two shear-thinning fluids with different flux indexes. The Newtonian fluid (from now on referred to as fluid $\mathrm{N}$ ) is a solution of $20 \%$ wt glycerol (Glycerol $>99 \%$ FCC, supplied by Sigma-Aldrich, UK) and $80 \%$ wt polyethylene glycol $M_{w}=400 \mathrm{Da}$ (PEG400, supplied by Sigma-Aldrich, UK). This mixture has a viscosity of $0.211 \mathrm{~Pa}$ s and was chosen to reduce the viscosity of glycerol and cover a wide range of the 


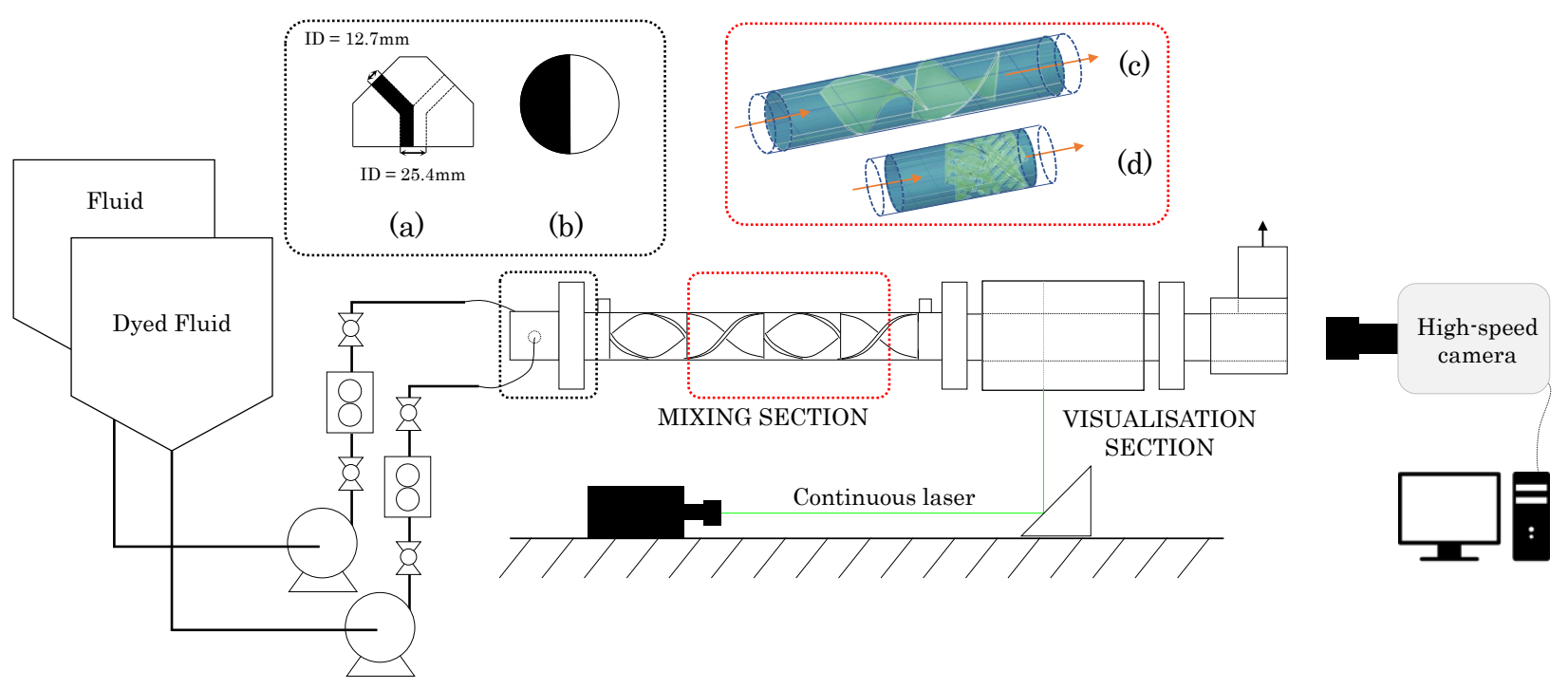

FIG. 1. Schematic of the experimental setup used for the PLIF measurements. The first inset shows (a) the top view of the Y-junction inlet and (b) the distribution of the streamlines in the cross-section as they enter the mixing section. The second inset shows a three-dimensional schematic of the housing of the mixing elements in the tube for (c) the Helical and (d) the SMB-R geometry. The orange arrows show the flow direction. Further details of the mixing elements characteristics are provided in SI-1.

Reynolds number (Re) for the Newtonian benchmark investigations, whilst maintaining a refractive index close to that of PMMA $\left(R I_{P M M A}=1.4905, R I_{N}=1.47\right)$ to optimise the clarity of the images. The Boger fluid (fluid B) was obtained by adding 200ppm of polyacrylamide (PAA, $M_{w} \approx 5 \times 10^{6} \mathrm{Da}$, supplied by Sigma-Aldrich, UK) in pure glycerol, whilst the shear-thinning fluids (fluid ST1 and fluid ST2) were prepared by dissolving Xanthan Gum (XG, supplied by Sigma-Aldrich, UK) in water/glycerol solutions. Densities for all solutions were measured through a calibrated picnometer at room temperature. Specifications of the fluid compositions, densities and solvent viscosities are summarised in Table I. Note that the value measured for the solvent viscosity of the Boger fluid reveals that the precise composition of the glycerol used is around $98 \% \mathrm{wt}^{55}$.

Given the large volumes of solutions required (approximately $25 \mathrm{~L}$ of liquid per set of experiments with one type of mixer), polymers were added in stock solutions, which were subsequently diluted with the correct amount of glycerol. Specifically, for fluid B, a stock solution at 500ppm of PAA in glycerol was prepared by gently dispersing the PAA in glycerol. During this stage, glycerol was kept at a temperature of $50 \mathrm{C}$ to reduce its viscosity and facilitate polymer dissolution. During the dispersion step, it was important to form a swirling flow in the container and gradually pour the powder in the centre of the vortex to avoid agglomeration. Similarly, for the two shear-thinning fluids, the stock solutions were prepared by adding XG to warm milli-Q water (DW, temperature kept at $40 \mathrm{C}$ ) in the exact ratio required for the final solutions. The stock solutions were stirred gently with a magnetic stirrer for at least 24 hours to ensure full dispersion of the polymer powders. Since glycerol is used in all fluids investigated, a stock solution of Rhodamine 6G (supplied by Sigma-Aldrich,
UK) at a concentration of $10^{-6} \mathrm{~mol} / \mathrm{L}$ in pure glycerol was also prepared beforehand. The experimental fluids were finally obtained by adding the proper amount of glycerol (partially from the dyed stock to obtain the dyed fluid) to the stock solutions. The final batches were stirred for 12 hours with a three blades marine propeller at low rotational speed. Once they were fully homogenised, the fluids were loaded in the tanks of the setup and left to rest at least for 3 days. To check the consistency in the composition of the experimental fluids, densities and viscosity measurements were performed before and after each experiment with few samples of fluids, showing no effect of polymer degradation.

\section{Rheological characterisation}

The rheological properties of all the fluids were measured in an Anton Paar MCR302 stress-controlled rotational rheometer, equipped with a Peltier plate to precisely control the operating temperature and a cone-plate geometry (OD: $50 \mathrm{~mm}$ and truncation angle of 1). For all measurements, sample menisci were sealed with silicon oil $\left(4.5 * 10^{-3} \mathrm{~Pa} \mathrm{~s}\right)$ to avoid solvent evaporation. Steady-shear flow tests were performed to obtain the flow curves of all the experimental fluids at $21 \mathrm{C}$. The tests were performed by ramping up the shear rate $\dot{\gamma}$ from 0.01 to $1000 \mathrm{1} / \mathrm{s}$. Viscosity values at different shear rates are presented for all the viscoelastic solutions in Fig. 2. As can be observed, the Boger fluid maintains a constant viscosity throughout the range of shear rates investigated (average value equal to $0.943 \pm 0.025 \mathrm{~Pa}$ s), whilst the two XG solutions present a shear-thinning behaviour. Note that the change in the solvent compositions of the XG solutions was forced by the need to obtain a strongly pronounced shear-thinning behaviour with- 
TABLE I. Composition, density and solvent viscosity $\eta_{s}$ of the test fluids. For all non-Newtonian fluids, $\eta_{s}$ is the viscosity of the solvent before polymer addition

\begin{tabular}{|c|c|c|c|c|c|c|c|}
\hline & Glycerol (\% wt) & PEG400 (\% wt) & $\mathrm{DW}(\% \mathrm{wt})$ & PAA (\% wt) & $\mathrm{XG}(\% \mathrm{wt})$ & $\rho\left(\mathrm{g} \mathrm{cm}^{-3}\right)$ & $\eta_{s}(\mathrm{~Pa} \mathrm{~s})$ \\
\hline Fluid N & 20 & 80 & - & - & - & 1.133 & 0.211 \\
\hline Fluid B & $100 *$ & - & - & 0.02 & - & 1.258 & 0.854 \\
\hline Fluid ST1 & 90 & - & 10 & - & 0.05 & 1.237 & 0.21 \\
\hline Fluid ST2 & 70 & - & 30 & - & 0.2 & 1.18 & 0.022 \\
\hline
\end{tabular}

out reaching excessive values of viscosity in the lower range of shear rates. Both shear-thinning viscosities can be fitted with the Carreau-Bird model:

$$
\eta=\eta_{\infty}+\left(\eta_{0}-\eta_{\infty}\right)\left[1+\left(\lambda_{c} \dot{\gamma}\right)^{2}\right]^{\frac{n-1}{2}}
$$

where $\eta_{0}$ is the zero-shear viscosity, $\eta_{\infty}$ is the infinite-shear viscosity, $\lambda_{c}$ is the Carreau time constant and $n$ is the flux index. The model fits the experimental data within an error of $2.5 \%$. As we can observe from Fig. 2, as the shear rate increases, the shear viscosity of the solutions reduces by about an order of magnitude for fluid ST1, and almost three orders of magnitude for fluid ST2, with flux index $n$ of 0.61 and 0.26 and time constant $\lambda_{c}$ of $11.9 \mathrm{~s}$ and $89.5 \mathrm{~s}$, respectively. The zero-shear viscosities $\eta_{0}$ are found to be equal to 2.3 and 47.6 $\mathrm{Pa}$ s, whilst the infinite-shear viscosities $\eta_{\infty}$ are close to the solvents viscosities with values of 0.156 and $0.049 \mathrm{~Pa} \mathrm{~s}$ for fluids ST1 and ST2, respectively.

Steady-shear measurements were repeated in a limited range of shear rates $(\dot{\gamma}=0.1-1001 / \mathrm{s})$ to obtain the first normal stress difference of the three solutions. The range of shear rates applicable was limited on the lower limit by the sensitivity of the instrument (which for the normal force is typically around $10^{-2} \mathrm{~N}$ ) and on the upper limit by the onset of elastic instabilities ${ }^{30}$. Therefore, to overcome these limits, the tests were performed in a range of temperatures from 0 to $60 \mathrm{C}$ and the time-temperature superposition principle ${ }^{26}$ was used to obtain master curves spanning a wider range of shear rates. Fig. 3(a) reports the master curves at $21 \mathrm{C}$ obtained for the first normal stress difference $N_{1}$, the first normal stress coefficient $\Psi_{1}$, and the shear stress $\sigma$ as functions of the reduced shear rate $\left(a_{T} \dot{\gamma}\right)$, where $a_{T}$ is the temperature shift factor along the horizontal axis, used to collapse all the results obtained at different temperatures.

For all solutions the first normal stress difference is higher than the shear stress in the range of shear rates investigated, thus highlighting the significant elastic character of the solutions. For both shear-thinning solutions (second and third graph from the top in Fig. 3(a)), the slope of $N_{1}$ is lower than 2 (approximately equal to 0.64 ) and $\Psi_{1}$ presents a significant shear-thinning character throughout the range tested. The PAA solution (fluid B) also presents a slope of $N_{1}$ lower than 2 (around 1.3) and shear-thinning behaviour of $\Psi_{1}$, even if a small change of slope seems to appear below a shear rate of $10 \mathrm{l} / \mathrm{s}$. This trend possibly hints at the formation of a plateau of $\Psi_{1}$, which is predicted for Boger fluids at the limit of zero-shear by all constitutive models available in the literature $26,56,57$
The pronounced elastic character of all viscoelastic solutions highlight that the use of the Carreau-Bird model, mentioned above, can simply describe the shear-thinning character of the viscosity for fluids ST1 and ST2 but cannot properly describe the rheology of these solutions, which would require the use of more complex nonlinear models. The behaviour of $N_{1}$ indicates a deviation from what is predicted by models such as the upper-convective Maxwell and Oldroyd-B for all fluids considered. This means that the relaxation times of the solutions cannot be retrieved from this data by simple extrapolation but models that predict more complex behaviour of the first normal stress difference would be required. Given the limited range of data available to properly fit a nonlinear constitutive model able to predict also the shear-thinning behaviour, small oscillatory amplitude sweep (SOAS) tests were performed at multiple temperatures to obtain the viscoelastic response in the limit of linear deformations. Master curves at $21 \mathrm{C}$ of the storage $G^{\prime}$ and loss $G^{\prime \prime}$ moduli are presented for the three solutions in Fig. 3(b). Note that, since the concentration of PAA in fluid B is below the overlapping concentration $c^{*}$ (see SI-3 for details), the solution can be regarded as diluted and the values of the loss modulus are presented by subtracting the solvent contribution to the original values of $G^{\prime \prime}$ detected by the instrument (i.e., $\left.G_{r}^{\prime \prime} \equiv G^{\prime \prime}-\eta_{s} \omega\right)$. The same applies to fluid ST1, even though in this case the XG concentration is slightly above $c^{*}$, hence these solutions should be considered as semi-diluted ${ }^{26,58}$. In all three cases, the trends of $G^{\prime}$ and $G^{\prime \prime}$ clearly deviate from a single-mode Maxwell fluid, and the solutions are characterised by a spectrum of relaxation times, which can be obtained by fitting the experimental data with a constitutive model. Since in the limit of infinitesimal deformations all constitutive equations implemented to model the polymer contribution to the fluid stress simplify to the simple Maxwell model ${ }^{26,56}$, the experimental data obtained for the three fluids were fitted with the generalised multimode Maxwell equation, which yields the following relations for $G^{\prime}$ and $G^{\prime \prime}$ :

$$
G^{\prime}=\sum_{k=1}^{N} \frac{\eta_{k} \lambda_{k} \omega^{2}}{1+\left(\lambda_{k} \omega\right)^{2}}, G^{\prime \prime}=\sum_{k=1}^{N} \frac{\eta_{k} \lambda_{k} \omega}{1+\left(\lambda_{k} \omega\right)^{2}}
$$

where $\eta_{k}$ and $\lambda_{k}$ are the viscous constant and the relaxation time of the k-th mode, respectively, $\omega$ is the frequency of the sinusoidal oscillation and $N$ is the number of modes implemented to fit the data. Results of the fitting are shown by the continuous lines in Fig. 3(b) and fitting parameters are summarised in Table II. Finally, the characteristic relaxation times of the three solutions were obtained as weighted averages of 


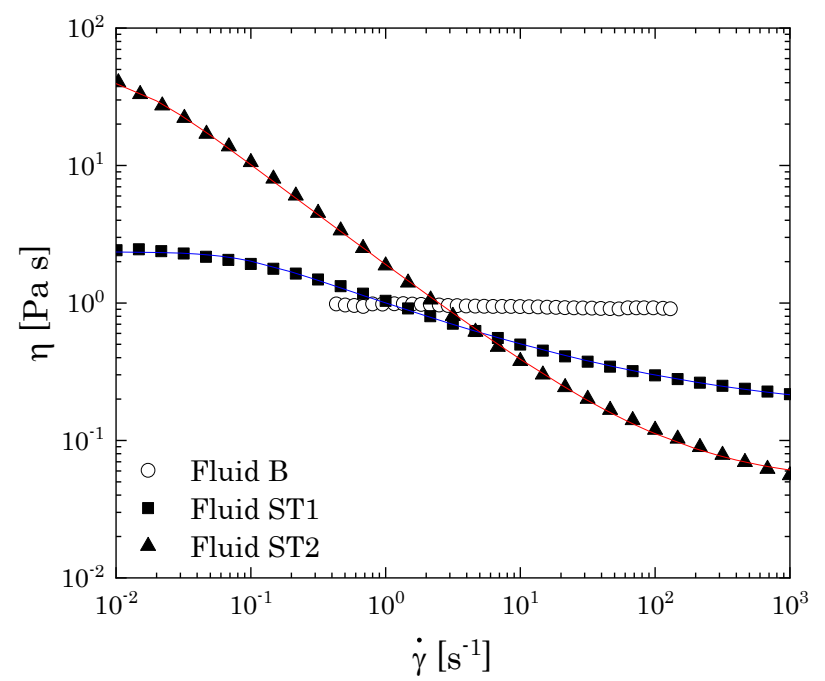

FIG. 2. Steady-shear viscosity of the viscoelastic solution obtained at $21 \mathrm{C}$.

each single mode ${ }^{39}$ :

$$
\lambda=\frac{\sum_{k=1}^{N} \lambda_{k} \eta_{k}}{\sum_{k=1}^{N} \eta_{k}}
$$

yielding values of $5.5 \mathrm{~s}, 15 \mathrm{~s}$ and $38 \mathrm{~s}$ for fluids B, ST1 and ST2, respectively.

\section{E. Relevant dimensionless numbers}

To obtain a proper comparison between the investigations performed with different solutions, the relevant dimensionless numbers should be specified. Since all experiment were conducted at various flow rates and with different fluid properties, the first dimensionless number to consider is the Reynolds number Re, which indicates the contribution of the inertial to the viscous forces in the flow and for both geometries can be defined as a modified version of the typical relation used for pipes $^{54}$ :

$$
R e \equiv \frac{\rho \frac{U}{\varepsilon} D}{\eta_{a}}
$$

where, $\rho$ is the fluid density, $U$ is the average fluid velocity evaluated considering an empty pipe of diameter $D, \varepsilon$ is the porosity of the mixing elements and $\eta_{a}$ is the fluid viscosity for fluids $\mathrm{N}$ and $\mathrm{B}$, whilst for the two shear-thinning fluids it can be more generally defined as the fluid viscosity at the apparent shear rate in the mixer $\dot{\gamma}_{a}$ (where $\dot{\gamma}_{a}$ is evaluated as $\left.8 U / \varepsilon D^{59}\right)$. The relevance of the elastic contribution is quantified through two different dimensionless numbers, the Deborah number De and the Weissenberg number Wi. The Deborah number, defined as the ratio between the relaxation time and a characteristic time scale of the deformation in the flow ${ }^{26,60}$, indicates whether or not the polymer chains are completely relaxed in the flow, hence inducing a fluid-like or a solid-like response of the fluid to the kinematics imposed by the base flow. In our case, we consider as the characteristic flow time $t_{f}$ the residence time in the single mixing element, which is related to the frequency with which the flow is periodically re-oriented during the mixing process. De can be defined as follows:

$$
D e \equiv \frac{\lambda}{t_{f}}=\frac{\lambda U}{L \varepsilon}
$$

The Weissenberg number is defined as the ratio between the fluid relaxation time and the reciprocal of the shear rate scale and can be interpreted as the ratio of the elastic to viscous forces ${ }^{60}$. Hence, we can take:

$$
W i \equiv \lambda \dot{\gamma}_{a}=\frac{8 \lambda U}{D \varepsilon}
$$

In our case, the two numbers differ only by a geometric parameter, the mixing element aspect ratio (i.e., $A R=L / D$ ). In conditions where the Reynolds number is not negligible Wi and Re can be coupled in a fourth dimensionless number, the Elastic number El, defined as the ratio between the elastic and the inertial forces:

$$
E l \equiv \frac{W i}{R e}=\frac{8 \lambda \eta_{a}}{\rho D^{2}}
$$

Finally, in the presence of complex flow kinematics, characterised by large streamline curvatures and local extensional components, polymer chains can be highly stretched, causing a local increase of the normal stresses along the streamline direction, which triggers the onset of elastic waves. If the characteristic velocity of the elastic waves is lower than that of the base flow, instabilities can propagate in the flow ${ }^{61,62}$. Hence, a viscoelastic Mach number Ma, defined as the ratio between the average speed of the flow and the elastic wave speed, should also be introduced to properly analyse the mixing patterns at different operating conditions:

$$
M a \equiv(U / \varepsilon) /\left(\eta_{a} / \lambda \rho\right)^{1 / 2}
$$

where $\left(\eta_{a} / \lambda \rho\right)^{1 / 2}$ is the shear wave speed in a viscoelastic fluid ${ }^{63}$.

\section{RESULTS AND DISCUSSION}

\section{A. Newtonian benchmark}

To provide a reference for the analysis with the polymeric solutions, we first present the typical mixing patterns obtained with both geometries in the case of a Newtonian fluid. To distinguish between purely inertial and elastic effects, we obtained concentration maps with the reference Newtonian fluids at various Reynolds numbers, covering the range of $\mathrm{Re}$ investigated with the viscoelastic fluids. This analysis is particularly important at the highest Reynolds number tested (i.e. 

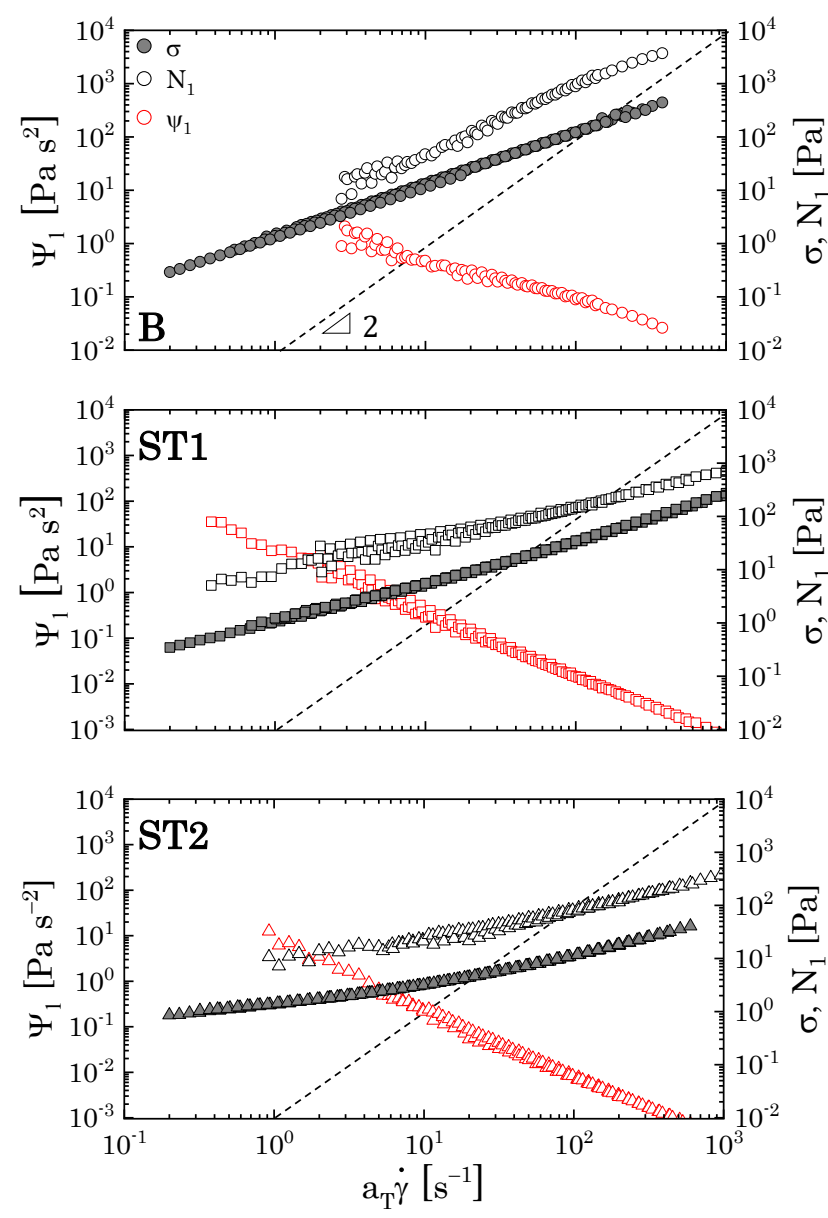

(a)
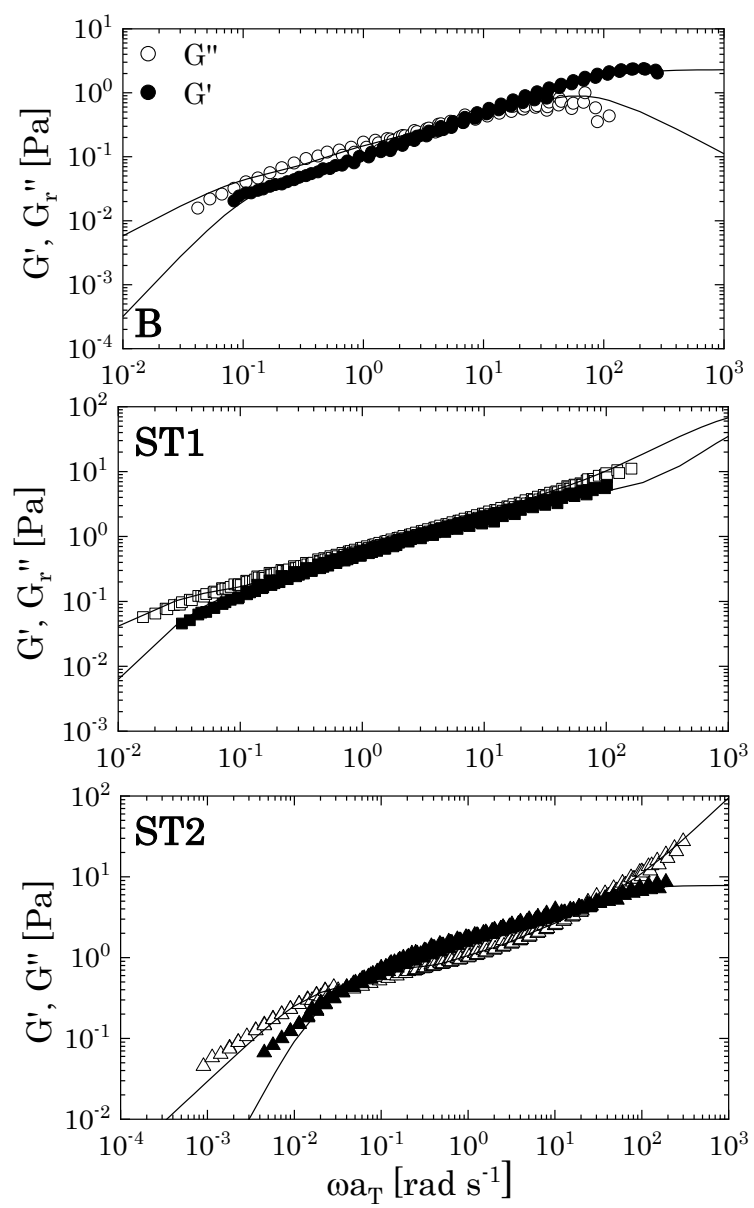

(b)

FIG. 3. (a) Master curves (at $21 \mathrm{C}$ ) of the shear stress, first normal stress difference and first normal stress coefficient against the reduced shear rate obtained from steady-shear tests and (b) master curves (at $21 \mathrm{C}$ ) of the loss (open symbols) and storage (closed symbols) moduli against the reduced oscillatory frequency obtained from SAOS tests for the three viscoelastic solutions. From top to bottom: Fluid B, Fluid ST1 and Fluid ST2. Note that the values of the loss modulus reported for fluid B and fluid ST1 correspond to the reduced loss modulus $G_{r}^{\prime \prime}$, obtained subtracting the solvent contribution.

$\operatorname{Re}=15)$, where secondary flows induced by purely inertial effects might start. For the standard helical geometry, the onset of secondary flows is generally reported to happen close to $R e=50$, even though the precise value can increase or decrease depending on the specific geometric features of the mixer (i.e. the ratio $L / D$, twisting angle and blade thickness) $)^{14,52,64,65}$. On the other hand, for the standard Sulzer SMX, which is the closest geometry to the SMB-R mixer employed in this study, the onset of secondary flows is generally expected at higher Reynolds numbers (i.e. Re $>100$ ), hence purely laminar regime is expected in all experimental conditions. Nonetheless, the standard concentration maps for this specific mixer design and their stability in the range of Re of interest are not available in the literature, necessitating their characterisation with a purely viscous fluid. In Fig. 4a the concentration maps after two and six helical mixer elements are reported for $0.6<\operatorname{Re}<15$. In all cases, the maps are perfectly stable in time, and no oscillations or vibrations are observed even at the highest Re. The mixing patterns are typical of the helical mixer ${ }^{9}$ and reflect the mixing mechanism induced by this specific geometry: as the fluid moves across the first element, the initial semicircles of dyed and undyed fluid are split in half, forming two streams, each composed at 50/50 of dyed and undyed liquid, which stretch separately along the twisted walls of the mixing element and then recombine at the end. This procedure is repeated as the fluid passes through the subsequent mixing elements, the striations are further split, stretched and recombined, creating a lamellar structure where elongated striations of dyed fluid are interleaved with ones of undyed fluid. Progressively the striation thickness decreases and the contact interfacial area between the two streams increases. In laminar conditions, the total number of striations for each liquid stream for a mixer with $N_{e}$ elements is theoretically predicted to be $2^{N_{e}}$, in the absence of molecular diffusion ${ }^{52}$. This prediction is recovered in the experimental results obtained after two mixer elements, where four stria- 
TABLE II. Fitting parameters of the multi-mode Maxwell model obtained from the experimental mastercurve of $G^{\prime}$ and $G^{\prime \prime}$ and final relaxation times evaluated using Eq. 5 for the three viscoelastic solutions

\begin{tabular}{lcccccccc}
\hline \hline & & 1 & 2 & 3 & 4 & 5 & 6 & $\lambda(\mathrm{s})$ \\
\hline \multirow{2}{*}{ Fluid B } & $\eta_{k}(\mathrm{Pas})$ & 0.025 & 0.047 & 0.123 & 0.388 & - & - & 5.5 \\
& $\lambda_{k}(\mathrm{~s})$ & 0.015 & 0.103 & 0.865 & 7.97 & - & - & 3.22 \\
\multirow{2}{*}{ Fluid ST1 } & $\eta_{k}$ (Pa s) & 0.032 & 0.06 & 0.082 & 0.23 & 0.65 & 1.35 \\
& $\lambda_{k}(\mathrm{~s})$ & $1.5 e^{-5}$ & $8.4 e^{-4}$ & 0.027 & 0.18 & 1.35 & 20 \\
\multirow{2}{*}{ Fluid ST2 } & $\eta_{k}$ (Pa s) & 0.095 & 0.074 & 0.25 & 0.81 & 5.7 & 22.53 & 38 \\
& $\lambda_{k}(\mathrm{~s})$ & $2.2 e^{-7}$ & 0.02 & 0.14 & 0.82 & 6.13 & 48.36 & \\
\hline \hline
\end{tabular}

tions are clearly distinguishable for each liquid stream (black and white striations), whilst after six elements the distinction between perfectly unmixed striations is blurred by the diffusion acting at the interface of the thinnest lamellae.

The effect of the Reynolds number on the mixing patterns is revealed only by the differences in the shape of the individual striations in terms of elongation, curvature and connectivity. As the mean velocity of the flow increases, the helicoidal curvature of the mixer walls induces a stronger radial component of the velocity field, which modifies the velocity profiles in the space between the pipe and the mixer walls, thus changing the stretching field and consequently the shape of the striations. This is confirmed by the analysis of the local velocity profiles obtained from 3D CFD simulations at different Reynolds numbers (for details on the CFD simulations refer to SI-4). Despite the differences observed, in the range of Re tested, the mixing mechanism and the mixing efficiency remain unchanged. To verify this, we calculated the coefficient of variance $(\mathrm{CoV})$ of the images as:

$$
\operatorname{CoV}=\frac{\sigma_{C}}{\bar{C}}=\frac{\sqrt{\frac{1}{N} \sum_{i=1}^{N}\left(C_{i}-\bar{C}\right)^{2}}}{\frac{1}{N} \sum_{i=1}^{N} C_{i}}
$$

where $\sigma_{C}$ is the standard deviation of the mass fraction $C$ in the whole cross-section, $\bar{C}$ is the average mass fraction of a perfectly mixed stream (i.e. 0.5 for all cases studied) and $N$ is the number of pixels in the image. Results, reported in Table III, show that the values for the different Re tested do not change significantly, as expected when the mixer performs in the fully laminar regime ${ }^{50}$.

Similarly to the helical mixer, the concentration maps obtained with the SMB-R design are perfectly stable in time in the whole range of Re investigated. Examples of the mixing patterns obtained after 1, 4 and 8 mixing elements are presented in Fig. 4(b) for two different values of Re. The topology of the striations is unaffected by an increase in Re: as the two streams pass through the sets of bars constituting the mixing element, the vertical interface between them is modified to form a complex pattern, consisting of four "flames" of dyed and undyed fluid (first row in Fig. 4(b)). The process is repeated at each passage into a mixing element, thus yielding a complex lamellar structure constituted by thin striations of dyed and undyed fluid, which progressively occupy the entire

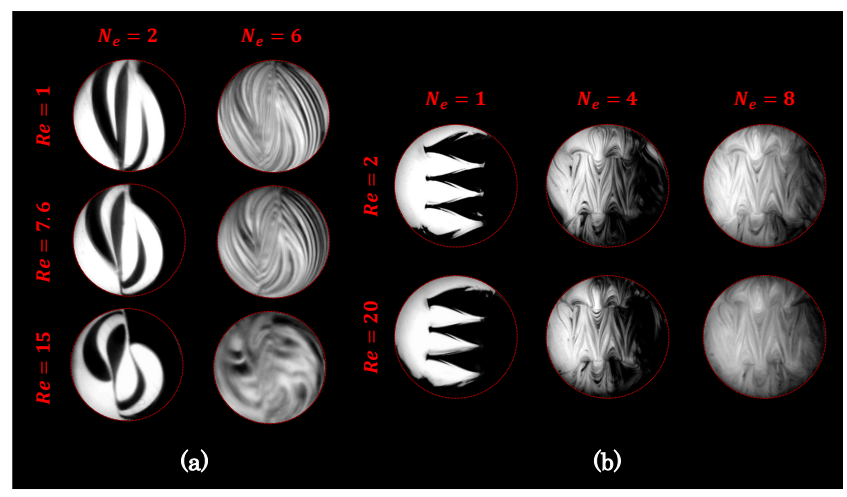

FIG. 4. Concentration maps obtained in the case of a Newtonian fluid (a) after 2 and 6 helical mixing elements at three different values of Re, and (b) after 1, 4 and 8 SMB-R mixing elements at $\mathrm{Re}=2$ and 20. The images presented were obtained from normalisation of the PLIF raw data.

cross-section of the pipe, as can be observed when comparing the maps obtained after 4 and 8 mixing elements. Up to the highest $\mathrm{Re}$ tested (i.e. $\mathrm{Re}=20$ ) the concentration maps maintain identical features, showing a great robustness in the mixing efficiency of this geometry in laminar conditions. The same qualitative behaviour is captured through 3D CFD simulations (SI-4.3), thus confirming the results obtained through the PLIF experiments. Values of the $\mathrm{CoV}$ are also reported in Table III for three different Re values.

\section{B. Mixing patterns with viscoelastic solutions}

When we move to the polymeric solutions, the mixing patterns for both geometries start to deviate from what was observed in the Newtonian cases as soon as De $>1$, independently of the specific fluid employed. Note that in all conditions tested $\mathrm{El}>50$, which indicates that all the irregularities that are described in the subsequent sections are dominated by elastic effects. Given the completely different flow fields induced by the two mixers, results will be shown separately for the two geometries. 
TABLE III. Experimental coefficient of variance $C o V$ (Eq. 11) obtained at all conditions investigated with the Newtonian fluid for both mixers.

\begin{tabular}{lcccccc}
\hline \multicolumn{1}{c}{$\operatorname{CoV}(-)$} & & $\mathrm{Re}=0.6$ & $\mathrm{Re}=2$ & $\mathrm{Re}=7.6$ & $\mathrm{Re}=15$ & $\mathrm{Re}=20$ \\
\hline \multirow{2}{*}{ Helical Mixer } & $N_{e}=2$ & 0.919 & 0.907 & 0.845 & 0.867 & - \\
& $N_{e}=6$ & 0.414 & 0.365 & 0.365 & 0.381 & 0.856 \\
& $N_{e}=1$ & - & 0.884 & 0.891 & - & 0.618 \\
\multirow{2}{*}{ SMB-R Mixer } & $N_{e}=4$ & - & 0.637 & 0.627 & - & 0.456 \\
& $N_{e}=8$ & - & 0.539 & 0.486 & - & \\
\hline \hline
\end{tabular}

\section{Helical mixer geometry}

Concentration maps after two mixing elements at different De are shown in Fig. 5 for the three fluids. Two different qualitative behaviours can be distinguished for all fluids above and below De $\approx 10$. For De $<10$, the splitting mechanism works and four striations per liquid stream can be distinguished, yet showing anomalous shapes and connectivity between them. Although altered compared to the elongated structures observed in the Newtonian case, dyed and undyed striations maintain symmetry between the two halves of the cross-section. As De is further increased, the number of striations reduces and for De $>10$ the initial streams are simply split in two striations with irregular shapes, completely losing any symmetry. These observations suggest that the splitting/stretching mechanism, on which the proper functioning of this mixer is based, is extremely sensitive to the elastic character of the fluid. For this geometry, at each passage in a mixing element, the base flow presents two critical points with significant change of the shear rate along the fluid pathlines and with curved streamlines: (i) the area of transition between two mixing elements, where a reorientation of the flow is induced by the alternating orientation of the elements, thus regulating the splitting of the streams, and (ii) the helicoidal curvature of the walls of the mixer, which induces the stretching of the striations. When the polymeric solutions are forced through the mixer, extra tensional stresses can develop in these regions, thus altering the stress distribution and consequently the shape of the velocity profiles, which in turn causes a change of the stretching field. Therefore, if the flow is slow enough to allow for the polymer chains to completely relax (De $<1$ ), the solutions behave as purely viscous liquids and the Newtonian concentration maps are preserved. On the other hand, if the characteristic time of the flow becomes smaller than the characteristic relaxation time $\lambda$, the polymer chains do not have time to fully recover their relaxed. This translates in a higher resistance to the stretching imposed by the base flow, causing the change in the shapes of the striations. As the flow rate is increased, the solid-like response of the fluid is enhanced and so is the resistance to splitting and stretching; the streams approach the behaviour of elastic "blobs", which are split at the edge of the first element, but flow through the subsequent one without stretching significantly (as can be observed by the more rounded shapes in Fig. 5 for $\mathrm{De}=44$ and $\mathrm{De}=83.8$ ).
The impact of the use of solutions with different rheological properties can be firstly observed in the diversity of the striations shapes, as highlighted by direct comparison of the maps obtained at De $\sim 3$ (first column in Fig. 5). At these intermediate values of De, the maps for each fluid are reproducible and steady, but the elongation of the striations varies with the fluid adopted. This difference suggests that fluids respond differently to the stretching imposed by the twisted walls, which implies that the specific shape assumed by the striations depends on the fluid extensional properties. Despite the lack of direct extensional measurements for the three fluids tested, general qualitative properties can be discussed based on data reported in the literature for similar solutions $\mathrm{s7}^{475}$. The major difference between the extensional properties of Polyacrylamide and Xanthan Gum solutions is related to the molecular structures of the two polymers. Polyacrylamide is a linear flexible polymer, as such, when in solution, PAA molecules assume a coiled configuration, whose response to extensional strains strongly depends on the rate at which the strain is applied, commonly showing a strain thickening behaviour. This means that the faster the deformation, the stronger the resistance of the polymer to extension (i.e. the extensional viscosity of PAA solutions increases with the extensional rate applied). On the other hand, Xanthan gum is typically described as a semi-rigid polymer ${ }^{66,67}$. Rigid and semi-rigid polymers align almost instantaneously with the flow field, thus presenting extensional properties that do not depend on the rate of deformation applied. For this reason, the extensional viscosity of XG solutions is usually reported to be constant with the extensional rate for a wide range of polymer concentrations ${ }^{47,58}$. Hence, as the mean velocity of the flow is increased, the PAA solution (i.e. Fluid B) shows an increased resistance to stretching compared to the XG solutions, and forms less elongated striations. In addition, because of the strain thickening character, Fluid $\mathrm{B}$ is also more sensitive to local variations of the strain and the stress fields, thus facilitating the onset of time-dependent instabilities in the base flow, which are instead delayed or suppressed in the case of solutions with uniform extensional properties $^{47,68}$

Fluctuations of the concentration maps are indeed observed in the case of Fluid B for De $>3$. Concerning the other two solutions, the onset of oscillations is delayed to higher De values (De $>12$ ) in the case of Fluid ST1, and does not occur at all in the whole range of flow rates tested (up to De $\sim 100$ ) in the case of Fluid ST2. In this time-dependent regime, different 


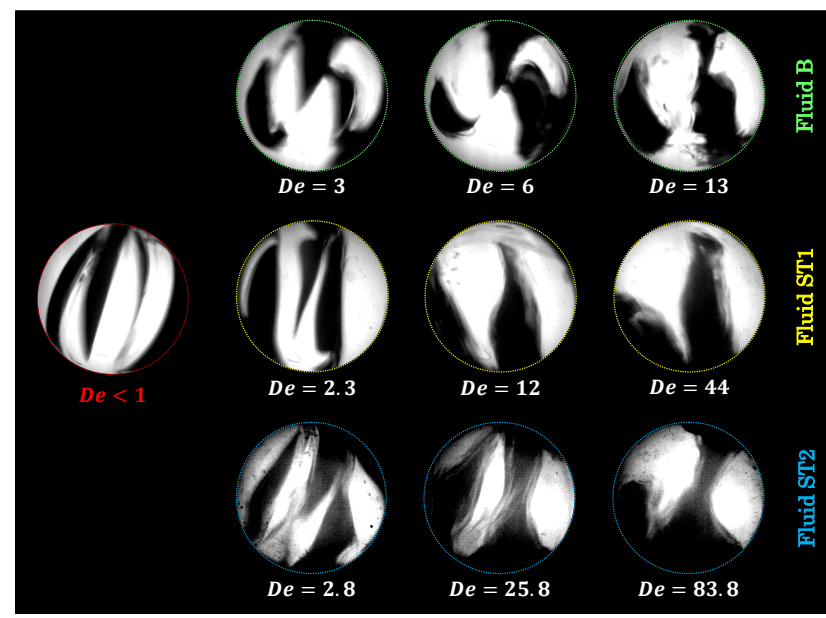

FIG. 5. Concentration maps after two helical mixing elements obtained at increasing values of De for the three viscoelastic solutions. From top to bottom: fluid B, fluid ST1 and fluid ST2.

striations preserve their main shape, yet showing fluctuation of their boundaries (original videos are available in the supplementary movie SM-1). To quantify the time-dependence of these oscillations, the mass fraction of dye was tracked in time for all the unsteady cases in random fixed positions at the test section cross sectional area, taken close to the striation boundaries. Examples of the time variations of the mass fraction obtained at increasing De for Fluid B are shown in Fig. 6a together with the reference signal for stable maps. Note that, since the total duration of the steady-state acquisition time $t_{a c q}$ changes with the operating flow rate (usually $t_{a c q}$ is at least three times higher than the mean residence time of the fluid from the entry at the first mixing element to the measuring plane position), the data are presented against a normalised time (i.e. $t / t_{a c q}$ ). In all cases, the acquisition frequency of the camera (i.e. $100 \mathrm{fps}$ ) was much higher than the highest frequency associated with the time variations of the concentration. The oscillations present strong irregularities and are non-periodic, as highlighted by the lack of distinctive peaks in the corresponding power spectral densities (PSD) reported in Fig. 6b. Nonetheless, for all conditions, the spectra present a power low decay of a few orders of magnitude, which is independent of the operating conditions. Such decay has been reported for both velocity and pressure drop fluctuations observed at the onset of elastic instabilities in different geometries ${ }^{37,48,69,70}$ and indicates that the flow field is characterised by a broad spectrum of timescales, which is usually associated with the concept of elastic turbulence ${ }^{44}$. For two helical elements, the average slope of the power law decay was found to be approximately 1.8 , independently of the operating flow rate and the specific fluid used. On the other hand, the amplitude of the oscillations, measured as the standard deviation of the mass fraction fluctuations $\left(S T D_{C}\right)$, slightly increases with De for both fluids, even if lower values are always observed for fluid ST1 compared to the Boger fluid.

These results suggest that the time-dependent behaviour of the concentration maps is strictly related to the flow distur- bance originating in the mixing area, as an effect of the interplay of the elastic behaviour of the solutions and the complex kinematics induced by the mixer geometry, and then propagates downstream of the mixing section. The strength of the perturbations depends on the elastic properties of the fluid, but its features are mainly controlled by the kinematics of the flow. In this event, the Mach number, as defined in Section 3.1 , is a better dimensionless quantity than De to individuate the onset of the time-dependent behaviour $37,61,62$, which should be expected only when the characteristic velocity of the base flow is higher than the velocity of propagation of the elastic wave (i.e. for $\mathrm{Ma}>1$ ). For fluid $\mathrm{B}$, the fluctuations start as soon as Ma becomes higher than 1, whilst for fluid ST1 the transition is delayed to $\mathrm{Ma}>5$.

As the number of elements is increased, the different transitions described above for two mixing elements still apply. For $1<\mathrm{De}<10$ the splitting/stretching mechanism works with some modifications in the shapes of the final striations, whilst at higher De the initial undisturbed streams are only split at the first element, and then randomly reoriented as they flow downstream. Examples of maps obtained at increasing number of mixing elements for the three different ranges of De are reported in Fig.7. Hence, for this specific geometry, an increase of the mean flow rate, which entails an increase of the characteristic De, is detrimental for mixing purposes, independently of the number of mixing elements used. The onset of the time-dependent behaviour also remains the same. The oscillations still present a strong chaotic character, which does not change significantly with the number of mixing elements: the PSD profiles do not present any significant peaks and decay following a power law with slope close to 2 , whilst the amplitude of the oscillations slightly increases when moving from two to four mixing elements but remains the same as the number of elements is further increased, revealing also less sensitivity to an increment of De.

A summary of the power law indexes of the PSD signals and amplitude of oscillations for all time-dependent conditions, obtained through a statistical analysis of the fluctuations in 50 random positions of the maps, is presented in Fig. $6 \mathrm{c}-\mathrm{d}$. Note that, even if locally the strength of the oscillations is significant, variations of the mass fraction obtained as a mean over the whole cross-section are almost negligible (Fig. 6e). This suggests that the scale of the perturbations remains small compared to the whole pipe cross-section. The disturbances maintain their localised character without amplifying their effects at the macroscopic level. Hence, even if the local oscillations present the characteristics associated with elastic turbulence, the effects at the large scale are negligible and the mixing does not benefit from an increase in the elastic character of the solutions as it is usually observed in microscale applications at the onset of elastic turbulence ${ }^{71,72}$.

\section{2. $S M B-R$ geometry}

Similarly to the helical geometry, deviations in the main features of the mixing patterns from the Newtonian case are observed as soon as De $>1$, yet showing a more complex 

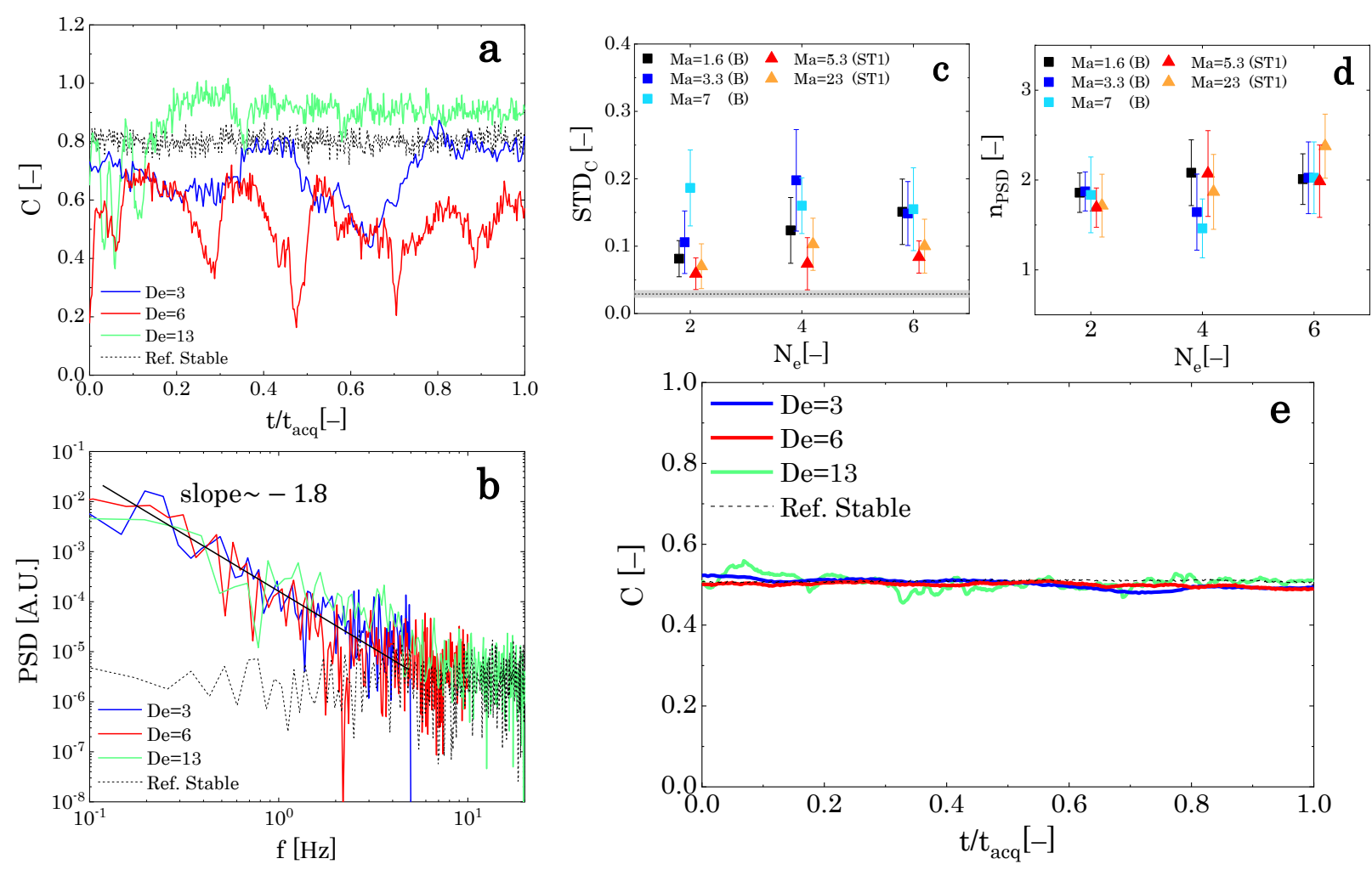

FIG. 6. (a) Example of local time variation of the dye mass fraction at increasing De, for fluid B. The black dotted signal represents the reference taken at $\mathrm{De}<1$, when the behaviour is assumed to be Newtonian; (b) corresponding power spectral density (PSD) functions; (c) amplitudes of the local oscillations, evaluated as standard deviation of the signals and (d) slope of the power law decay of the corresponding PSD functions for all unsteady conditions. For each condition, the points reported were obtained by averaging the values over 50 random positions taken close to the striations boundaries (the error bars represent the variation in STDc between the 50 points sampled). The black dashed line in panel (c) is the reference value in stable conditions (the grey band is the variation in $S T D_{C}$ between 50 random positions). The data points associated to the number of mixing element $N_{e}$ in both panel (c) and (d) are not vertically alligned for visual purposes; (e) example of the time variation of the average mass fraction across the whole cross-section for the same conditions reported in panel (a).

time-dependent behaviour compared to the previous case, which is influenced by the type of fluid and the number of mixing elements. Findings obtained after only one mixing element are first discussed. Before the onset of any timedependent behaviour, independently of the fluid used, the maps present stable patterns, which partially resemble the Newtonian case, however showing a less elongated and symmetric topology (see SI-5.1). This behaviour, in analogy with what was previously observed in the helical geometry, can be associated with the extra tension developed in the direction of the streamlines, which acts against the proper stretching of the fluid streams, thus altering the final shape of the striations. As for the previous geometry, in the case of the Boger fluid (fluid B) the unsteady behaviour starts at $\mathrm{Ma}>1$ : the striations present a more elongated and irregular aspect and vibrate preserving their original shape. The PSD signal associated with the local oscillations decays with a power law of slope 2 , as was also found in the other geometry (see SI-5.2). As the flow rate is increased and a Ma higher than 10 is reached $(\mathrm{Ma} \approx 12)$, the maps assume a more elongated configuration and the fluctuations become stronger (Fig. 8a). The PDS sig- nal presents a two-step power law decay, characterised by two different slopes, one in the lowest range of frequencies, close to 0.5 , and the other at intermediate frequencies, with values between 2 and 3 (Fig. 8b). A similar trend has been observed in the PSD signals of the pressure drop fluctuations associated with the flow of viscoelastic solutions across an array of obstacles ${ }^{37}$. The authors linked the two-step decay to the superposition of two different instabilities, one related to the wash out of small volume of fluids, formed upstream of the obstacles, and the other related to the presence of elastic turbulence. In this case, even though direct observations of the flow are not available in the mixing area, a similar mechanism might be in place: in the direction of the flow, the bars constituting the mixing elements can be seen as a complex array of rectangular obstacles; small recirculating zones can form upstream the bars and subsequently destabilise, thus generating the strong fluctuations observed in this regime.

In the case of fluid ST1, the transitions observed at increasing Ma differ from those depicted above: an intermediate regime, characterised by small vibration of the concentration profiles is not observed, but instead strong time-dependent 


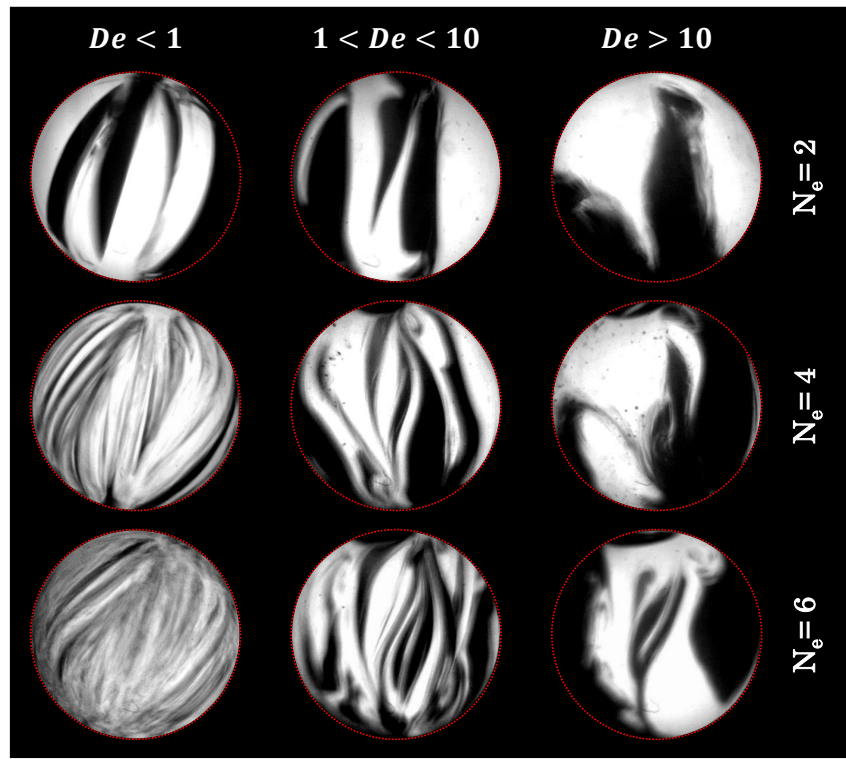

FIG. 7. Evolution of the concentration maps with the addition of mixing element in the helical geometry for the three different regimes individuated.

fluctuations appear even at the lowest flow rate tested (corresponding to $\mathrm{Ma}=0.3$ ). The shape of the maps changes significantly in time, assuming a quasi-periodic character with similar patterns that repeat continuously in time. The periodic character can be captured in some key locations, through the time variation of the concentration profiles, but it does not extend to the whole cross-section. Examples of the quasiperiodic oscillations captured for $\mathrm{Ma}=0.3$ and $\mathrm{Ma}=0.86$ are reported in Fig. 9a together with the corresponding PSD signals (Fig. 9b), which show a small peak indicating the characteristic frequency of the oscillations. As the mean flow rate is increased, the striations assume more elongated shapes, and fluctuate vigorously in the whole cross-section, progressively gaining a stronger chaotic character and losing the local periodic character. As for fluid B, the corresponding PSD signals present a two-step power law decay, characterised by the same two different slopes (see SI-5.3), with no distinct peak identifiable. Similarly to what has been described for fluid B, the PSD double decay suggests that what is observed downstream can be related to the superposition of two instabilities: (i) localised releases of pockets of fluids, or 'dead zones', formed upstream the mixer element, which dominate in the low frequency regime, and (ii) purely elastic instabilities, which decay in a wide range of frequencies. With an increase of the shear-thinning character, the onset of a time-dependent behaviour is delayed to $\mathrm{Ma}>7$. A further difference from the previous cases is observed for fluid ST2: at Ma $=7.3$ the concentration in the cross-section does not oscillate continuously but instead shows a sudden change in topology, from one stationary pattern to another. As $\mathrm{Ma}$ is increased, strong fluctuations arise, presenting the same chaotic character found in the other two cases (refer to SM.2 for videos of all cases described).

The different behaviours observed might be related to the changes induced by different rheological properties to the formation and stability of the dead zones upstream of the mixing elements and can be rationalised by drawing on what has already been observed experimentally for flows past arrays of obstacles. In the case of shear-thinning fluids, the formation of dead zone can be initiated at $\mathrm{Ma} \ll 1$. This condition is identified as a 'dead-zone instability' ${ }^{37}$. At low flow rates, a small dead-zone first forms upstream, then grows in the axial direction opposite to the direction of the flow. As it grows, the dead zone is destabilised by the continuous flow and starts to wobble perpendicularly to the flow direction until it detaches from the obstacle and gets washed out with the main flow. The initial mechanism of formation of the dead zones is comparable to a shear banding phenomenon, where local regions at low shear rate form close to the upstream fixed wall of the obstacle, locally increasing the viscosity and thus creating discontinued regions of low (close to upstream wall) and high mobility. As a consequence, the shear-thinning character of the solutions strongly influences the formation and stability of the dead zones, as reported by Kawale and co-workers ${ }^{37,73}$, who observed that highly shear thinning solutions form bigger and more stable dead zones, whilst when the shear thinning is suppressed the characteristic size of the dead zones also reduces drastically.

Hence, the results obtained with the shear thinning fluids suggest that in the case of the low shear thinning fluid, the dead zones form and destabilise at very low flow rates, thus showing quasi-periodic oscillations of the concentration at $\mathrm{Ma}<1$, whilst for the highly shear-thinning case, the dead zones probably still form at low flow rates, but grow in size before losing their stability at a higher mean flow velocity, thus showing stable concentration maps up to $\mathrm{Ma}>1$. Specifically, the sudden change observed at $\mathrm{Ma}=7.3 \mathrm{might}$ be due to the release of a large dead zone formed from merging of various smaller ones, which at some point is washed out by the main flow. As the flow rate is further increased, the stability of the dead zones reduces, local releases influence the stability of zones downstream, creating a ripple effect that translates into a chaotic behaviour of the concentration profiles, characterised by a PSD with a double power law decay.

On the other hand, in the case of the Boger fluid, the characteristics of the first unstable behaviour detected suggest that the initial instability is not related to formation of dead zones but rather to disturbances typical of pure elastic instabilities, which could originate also downstream of the obstacle. For the simplified case of the flow of Boger fluids past a confined cylinder ${ }^{61}$ or a linear array of cylinders ${ }^{62}$, a spatially and temporally unsteady flow is typically reported downstream of the obstacle as soon as $\mathrm{Ma} \sim 1$, this is then followed by the formation of an unsteady upstream vortex at higher Ma. Even though the geometry of our system is far more complicated, a similar mechanism could explain the evolution of the unsteadiness observed for the Boger fluid, where mild vibrations of the concentration maps are observed for $1<\mathrm{Ma}<10$, followed by strong chaotic fluctuations, which could be related to the presence of unsteady upstream vortexes that cause a chaotic behaviour comparable to that observed in the shearthinning cases, but different in its origin. 

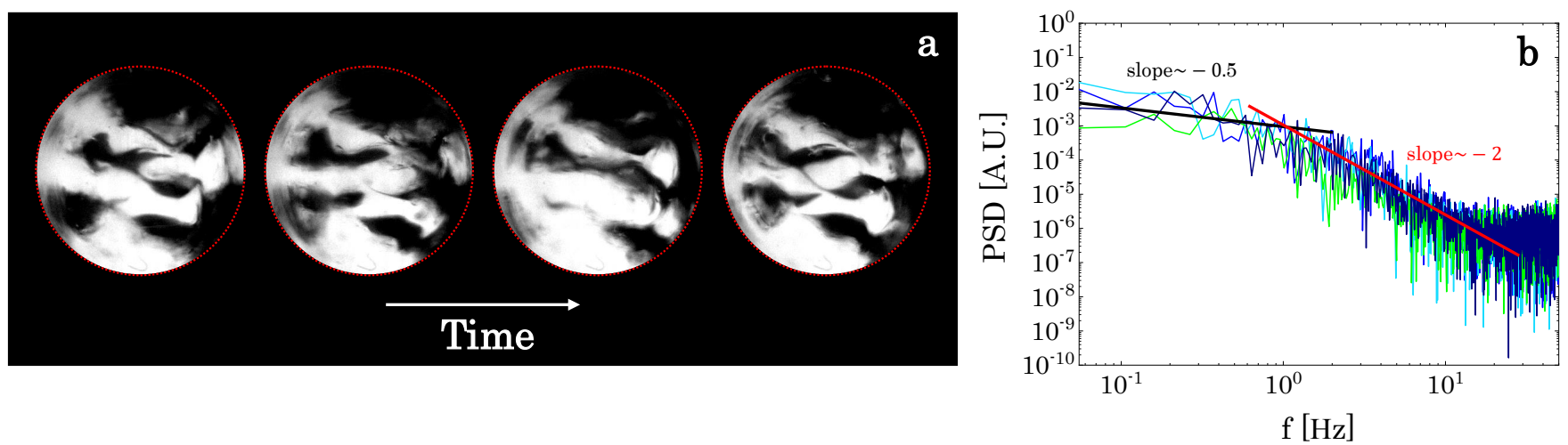

FIG. 8. (a) Time evolution of the concentration maps obtained at $\mathrm{Ma}=12$ with fluid $\mathrm{B}$ after one SMB-R mixing element (images are reported with a $\Delta t=2.5 \mathrm{~s}$ ); (b) corresponding PSD functions of the oscillations of dye mass fraction obtained in four random positions of the crosssection. The PSD shows a first power law decay (blue line, slope $\sim-0.5$ ) up to $f \approx 1 \mathrm{~Hz}$ and a second (red line, slope $\sim-2$ ), which extends in the higher range of frequencies.

The general features of the instabilities are preserved with the addition of mixing elements, yet showing small changes in the boundaries between the different behaviours described above. In the case of fluid ST1, the quasi-periodic character observed at the lowest flow rates is lost and the oscillations present a strong chaotic character in the whole range of conditions tested. Similarly, for fluid ST2, the unsteady behaviour still begins at $\mathrm{Ma}=7$, but the fluctuations are chaotic. These observations support the hypothesis of formation/wash out of dead zones: in the presence of further obstacles in the axial direction, the release of single pockets of fluid affects greatly the stability of those downstream, thus generating a ripple effect as soon as the dead zone get released. The topology of the maps is completely different than the one observed in the Newtonian case. The complex lamellar structure is substituted by more rounded irregular areas, which decrease in size with the further addition of mixing elements (Fig. 10). Nonetheless, as Ma increases, the characteristic dimension of the unmixed area gradually increases, becoming less sensitive to the number of mixing elements used (Fig. 10).

For the Boger fluid the first unstable behaviour, characterised by mild oscillations, is anticipated at $\mathrm{Ma}=0.4$, whilst strong chaotic oscillations appear at $\mathrm{Ma}=4$. As for the previous cases, the local disturbances generated in the first elements are progressively enhanced by the presence of further obstacles, thus shifting the onset of the instabilities to slightly lower values of Ma. The statistical values of the amplitude of the oscillations and the power law indexes of the PSD signals for all unsteady conditions found with the SMB-R geometry are summarised in Fig. 11. With one mixing element, since the fluctuations become progressively more chaotic and evenly distributed in the cross-section, the strength of the oscillations increases significantly with the increase of the mean flow rate, independently of the type of fluid, whilst showing a very mild increment with 4 and 8 mixing elements, where the fluctuations are less sensitive to a change in the mean flow velocity. By comparing the magnitude of the oscillations in Fig. 11 with those reported in Fig. 6c, the time-dependent behaviour in the SMB-R geometry is notably stronger than that
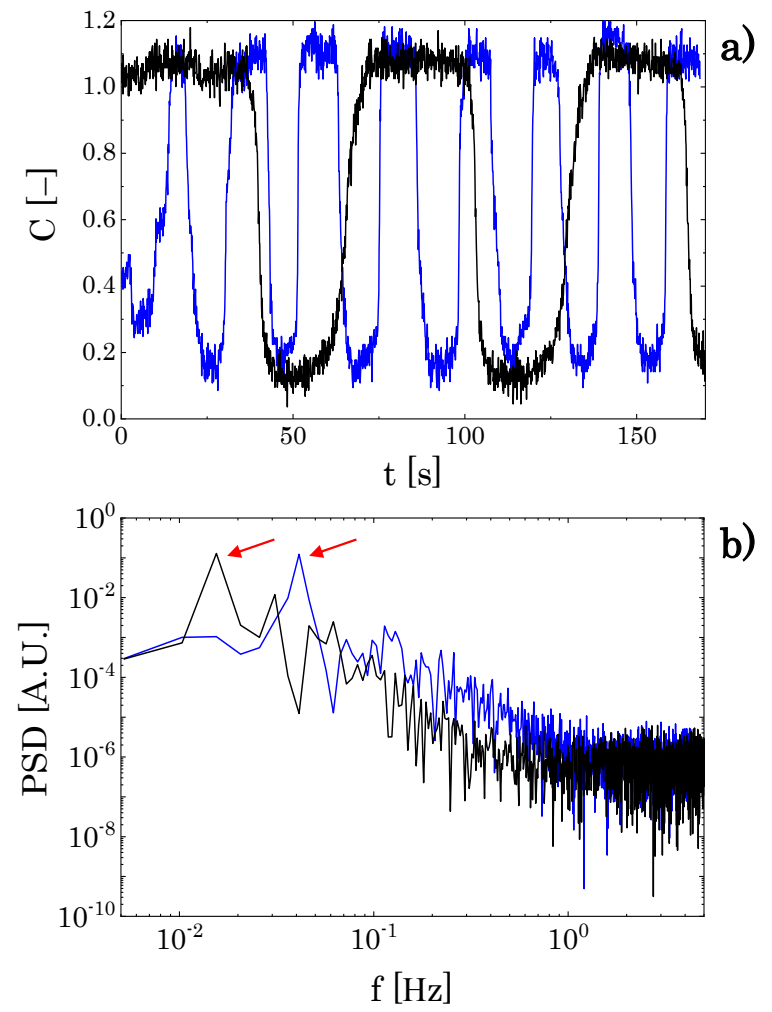

b)

FIG. 9. (a) Example of quasi-periodic fluctuactions observed in the case of fluid ST1 at low Ma after one SMB-R mixing element (black line: $\mathrm{Ma}=0.3$, blue line: $\mathrm{Ma}=0.86$ ); (b) corresponding PSD functions. The red arrows indicate the characteristic frequency of the oscillations.

in the helical mixer. This is further emphasised by the significant time variations found for the average mass fraction in all cases showing strong chaotic fluctuations (Fig. 12), thus highlighting the different nature of the instabilities observed in the two geometries. 


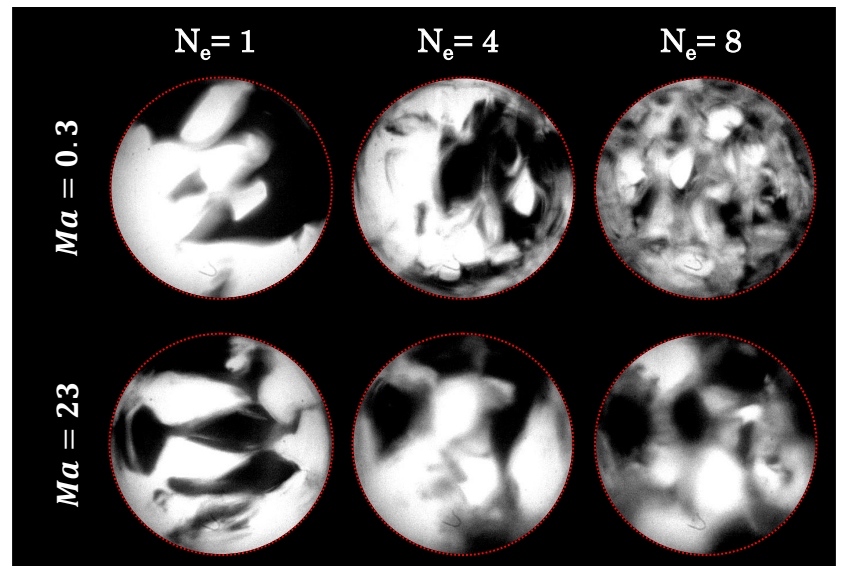

FIG. 10. Evolution of the concentration patterns across 1, 4 and 8 SMB-R mixing elements at increasing Ma (data obtained with fluid ST1). The images reported represent a single frame, since strong fluctuations of the concentration are observed for all the cases depicted.
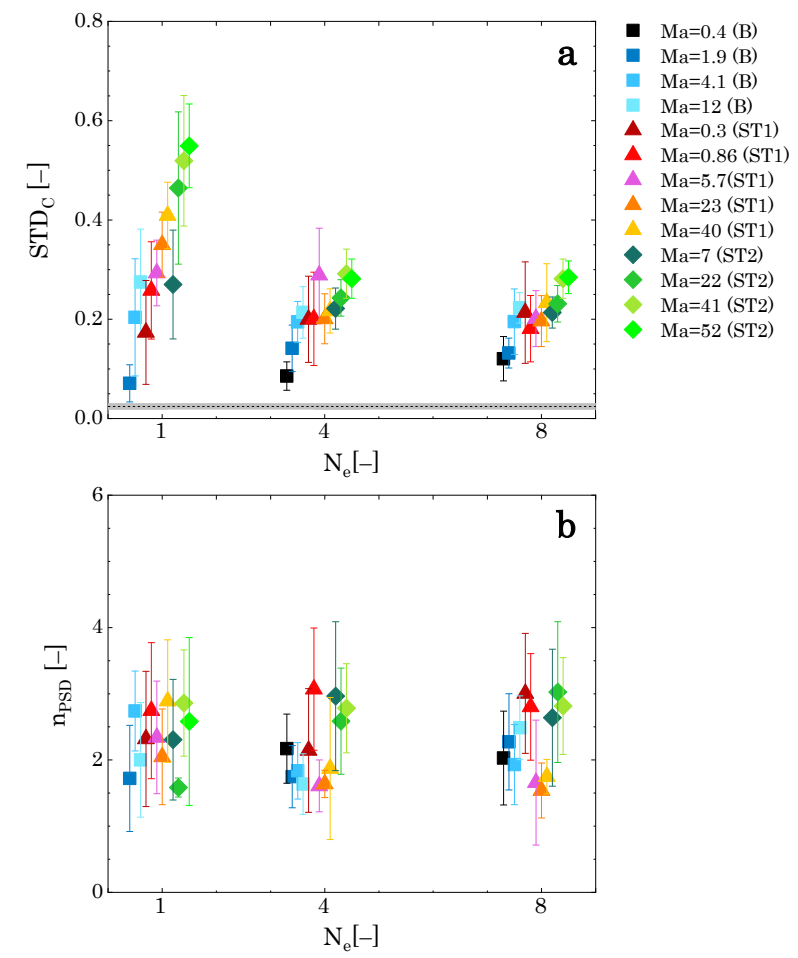

FIG. 11. (a) Amplitudes of the local oscillations, evaluated as standard deviation of the signals and (b) slope of the second power law decay of the corresponding PSD functions for all unsteady conditions in the SMB-R geometry. For each condition the points reported were obtained by averaging the values over 50 random point in the image (the errors bar represent the varition of $S T D_{C}$ between the 50 points sampled). The black dashed line in panel (a) is the reference value in stable conditions (the grey band is the varition of STDc between more than 50 random positions). The data points associated to the number of mixing element $N_{e}$ in both panels (a) and (b) are not vertically alligned for visual purposes.

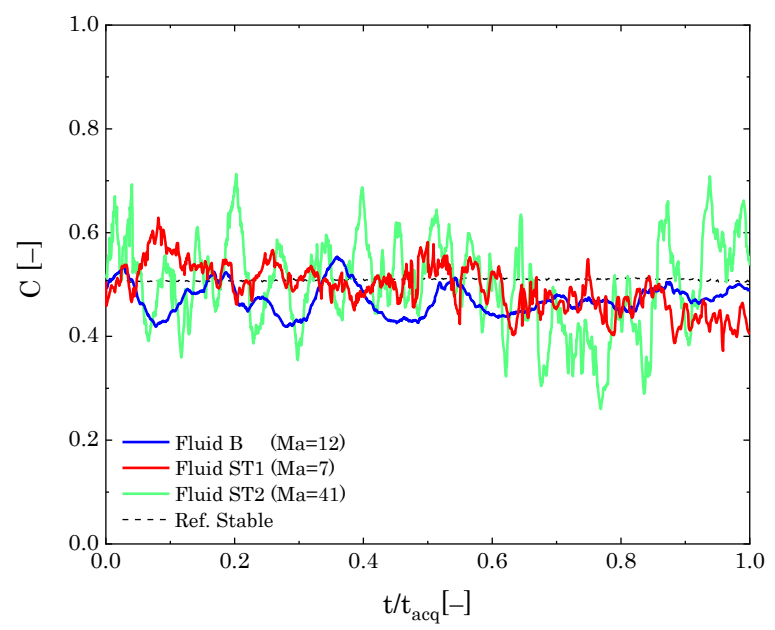

FIG. 12. Example of the time variation of the average dye mass fraction evaluated in the whole cross-section for three different conditions after 1 SMB-R element.

\section{Effects on the mixing efficiency}

A summary of the transitions described for both geometries and the resulting outcome in terms of mixing efficiency are reported in Fig. 13 and Fig. 14 for the Helical and the SMB-R mixers, respectively. In the case of the helical geometry, the same performances of the Newtonian case are observed only for De $<1$. A first deviation occurs at De $>1$ and low Ma, where the mixing patterns present an altered topology, characterised by less elongated striations. In this regime, the concentration maps are steady but the splitting/stretching mechanism is altered because of the more pronounced solidlike behaviour of the viscoelastic solutions, thus displaying a loss of mixing efficiency compared with the Newtonian case (expressed in terms of $\mathrm{CoV}$ ), which becomes evident as the number of mixing elements is increased. As De rises, the elastic character of the solutions is enhanced and the two initial streams remain completely unmixed. Nonetheless, as the mean flow velocity is increased, a time-dependent behaviour arises at $\mathrm{Ma}>1$. In this regime, the large unmixed areas start to fluctuate chaotically, still preserving their original shape. As can be seen from the Ma-El phase map in Fig. 13, the onset of the unsteady regime is influenced by the rheological properties of the fluid: as the shear-thinning character increases, the onset of the unsteady regime is pushed to higher Ma numbers and is completely suppressed for the highly shear-thinning fluid in the range of conditions tested. The cause of this stabilising effect could be attributed to two different aspects, one related to the more stable extensional properties of the semirigid Xanthan gum molecules, which infer a lower sensibility to the sudden reorientation of the flow forced by the geometry of the mixer ${ }^{47,68}$, and the other related to the possible suppression of the time-dependent instability at higher values of the Elastic number, which is typically observed in the case of elasto-inertial instabilities in different flows ${ }^{37,47,68}$. Fur- 

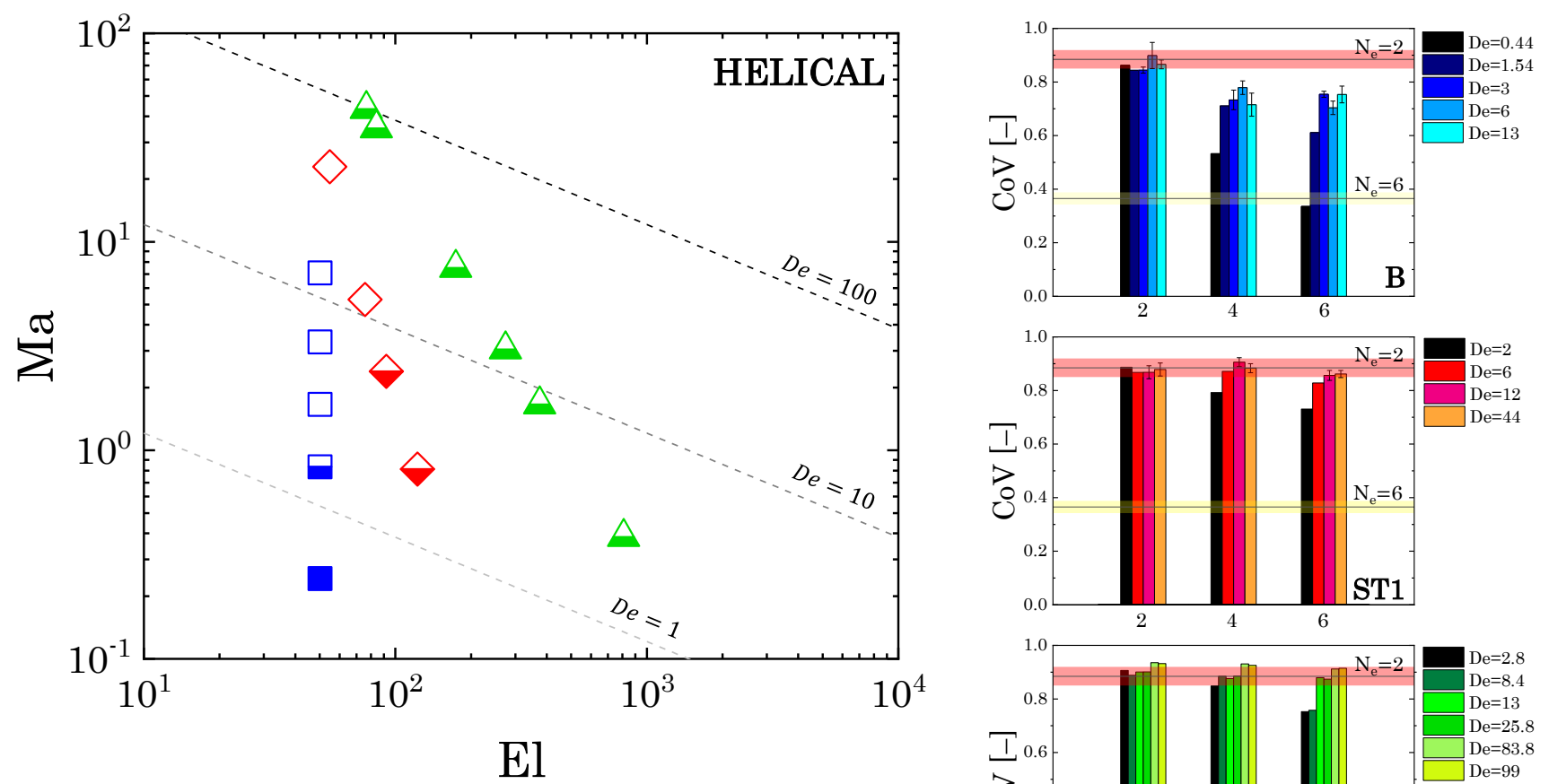

Newtonian-like (B)

Stationary (B)

Mild fluctuactions (B)
Stationary (ST1)

Mild fluctuactions (ST1)

Stationary (ST2)
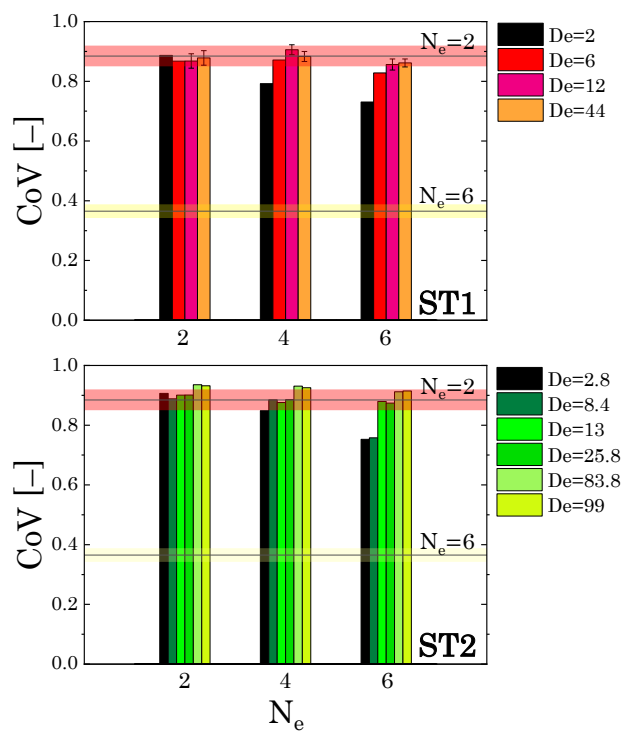

FIG. 13. On the left, Ma-El space phase map summarising the transitions observed in the Helical mixer (for all lengths studied); on the right, evolution of the mixing efficiency for all conditions tested. The coloured horizontal bands are reported for reference and represent the $\mathrm{CoV}$ values in the Newtonian case (the thickness of the band is the standard deviation over all the values obtained at different Re). The error bars represent the standard deviation of the $\mathrm{CoV}$ signals in the case of unsteady conditions.

ther experimental investigations of the flow field in the mixing area, performed with a wider range of fluid rheological properties, are required to clarify the nature of the instability; however, from a technological viewpoint, the effect of the onset of a time-dependent behaviour on the mixing efficiency of the helical mixer is negligible as the oscillations are localised at the boundaries of the striations and do not have the strength to amplify to the whole cross-section of the pipe. The mixing efficiency in this geometry is strictly dominated by the efficiency of the stretching/splitting mechanism, which is prevented by an increase of the elastic character of the solutions, thus showing a detrimental effect on the mixing efficiency as soon as De $>1$.

In terms of flow resistance, the onset of the instabilities does not strongly influence the magnitude of the friction factor and only a small increase can be observed at the highest Re tested (see Fig. 15a), although the power spectral densities of the pressure drop signals present a power law decay with a slope close to 2 . The trend remains the same in the whole range of Ma for which instabilities are observed, yet showing a progressive increase in the magnitude of the PSD and widening of the range of frequencies over which the decay extends.

For the SMB-R mixer, the transitions to different regimes are more complex and clearly influenced by the number of mixing elements and the rheology of the fluid. In the absence of shear-thinning (i.e. fluid B), the transitions observed are similar to the helical geometry: for $\mathrm{De}>1$ and $\mathrm{Ma}<1$, the shape of the concentration pattern is altered but the maps appear steady; as Ma exceeds unity, mild oscillations of the striations are observed; however, for this geometry, when Ma is further increased a different regime appears, characterised by more elongated striations that strongly oscillate in time. When the number of elements is increased, the qualitative behaviour persists but the boundaries between the unsteady transitions move to lower Ma. In the presence of a shear-thinning character, the phenomenology observed changes. For the mildly shear-thinning fluid (fluid ST1), strong oscillations are observed also at $\mathrm{Ma}<1$ : the oscillations appear quasi-periodic at the lowest flow rates but become progressively more chaotic as the mean flow velocity is increased. The same behaviour is observed for fluid ST2, with the only exception that the unsteady behaviour is delayed at higher values of Ma. The addition of mixing elements appears to simply enhance the chaotic character of the fluctuations.

The variety of the observations can be discussed assuming an analogy with the flow of viscoelastic solutions past arrays 

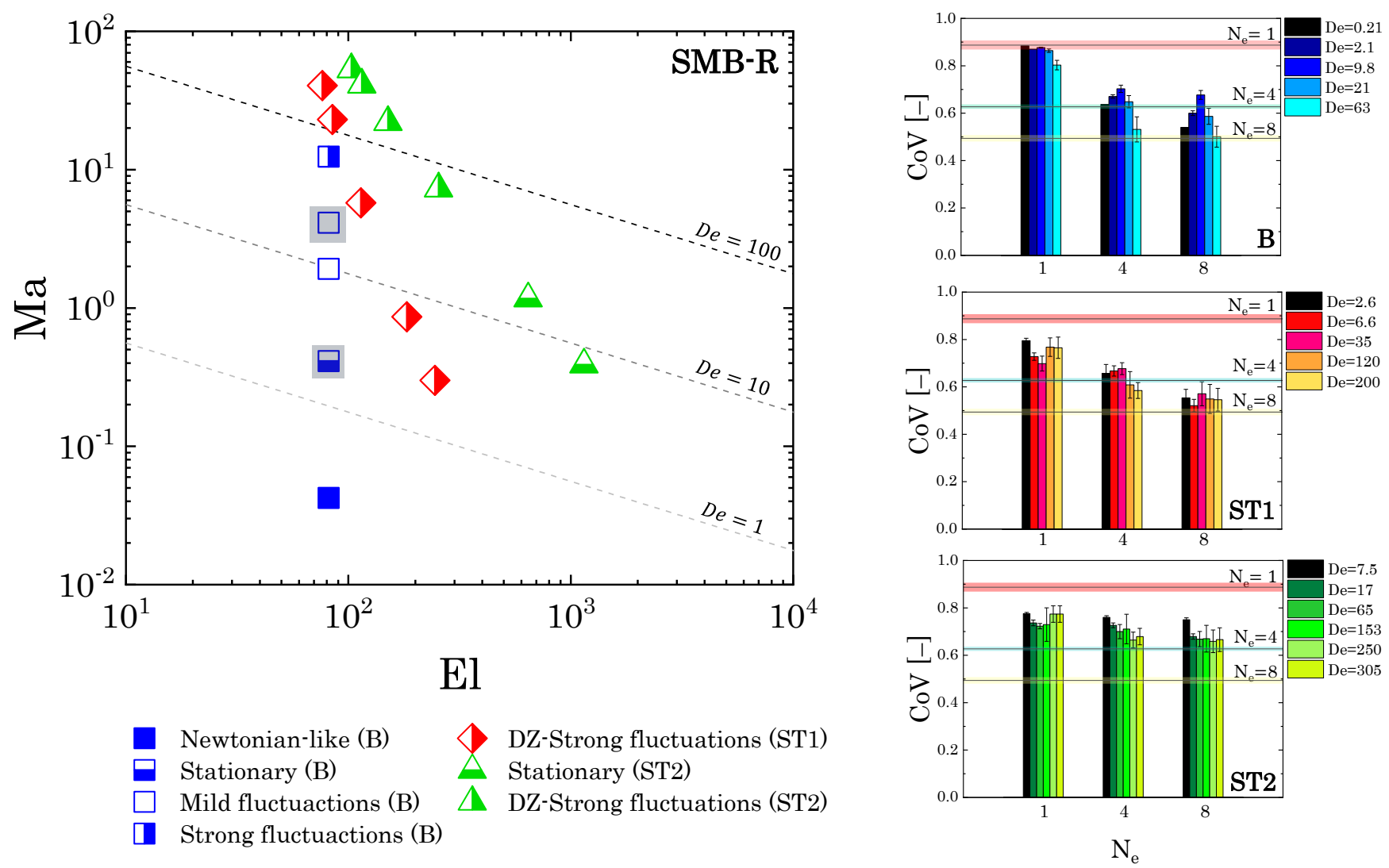

FIG. 14. On the left, Ma-El space phase map summarising the transitions observed in the SMB-R mixer (for all length studied). For fluid $\mathrm{B}$ the unstable behaviour is anticipated at $\mathrm{Ma}<1$ and the strong fluctuations appear at $\mathrm{Ma}<10$ when the number of mixing elements is increased (grey squares on the map); on the right, evolution of the mixing efficiency for all conditions tested. The coloured horizontal bands are reported for reference and represent the $\mathrm{CoV}$ values in the Newtonian case (the thickness of the band is the standard deviation over all the values obtained at different Re). The error bars represent the standard deviation of the $\mathrm{CoV}$ signals in the case of unsteady conditions.

of obstacles: in the case of the Boger fluid, the transitions observed suggest the presence of a first flow instability generated downstream of the mixing elements at lower Ma, followed by the formation of upstream vortexes ${ }^{62,74}$, which, given the complexity of the geometry, can interact and destabilise, generating strong fluctuations of the concentration. On the other hand, for the shear-thinning solutions, a dead zone can form upstream of the mixing element bars even at low Ma, which further grow in size up to the point where they lose their stability and get released ${ }^{37,73}$.

As opposed to the helical mixer, in the SMB-R mixer the effect of the onset of the instabilities on the mixing efficiency is not in all cases detrimental: the presence of strong fluctuations improves the mixing efficiency after only one mixing element, as can be seen in the bar charts in Fig. 14; however, as the number of elements is increased, the CoV does not always recover the same efficiency as the Newtonian case. Specifically, the formation of large dead zones, creates large unmixed areas, generating a negative impact on the mixing efficiency, as can be seen in the case of fluid ST2, where the $\mathrm{CoV}$ does not vary with the number of mixing elements. Additionally, even when the same efficiency of the Newtonian case is recovered, large temporal variations are still present, thus yielding uneven mixing performance. As regards the energy requirements, similarly to the helical mixer, the onset of the instability does not entail a significant increase in the magnitude of the flow resistance (Fig. 15b). The PSD functions of the pressure drop signal present the same qualitative behaviour observed in the other geometry, although showing a greater magnitude (i.e. at similar $\mathrm{Ma}$, the magnitude of the PSD values is almost four orders of magnitude higher for the SMB-R mixer than for the helical mixer), a finding that highlights the more pronounced strength of the chaotic fluctuations in the SMB-R mixer.

\section{CONCLUSIONS}

This work experimentally investigates the onset of viscoelastic instabilities in two continuous flow tubular static mixers and the subsequent effect on the topology of the mixing patterns. To address the influence of distinct geometric features on the onset and nature of the instabilities, two mixer geometries were selected, namely the Kenics helical mixer 

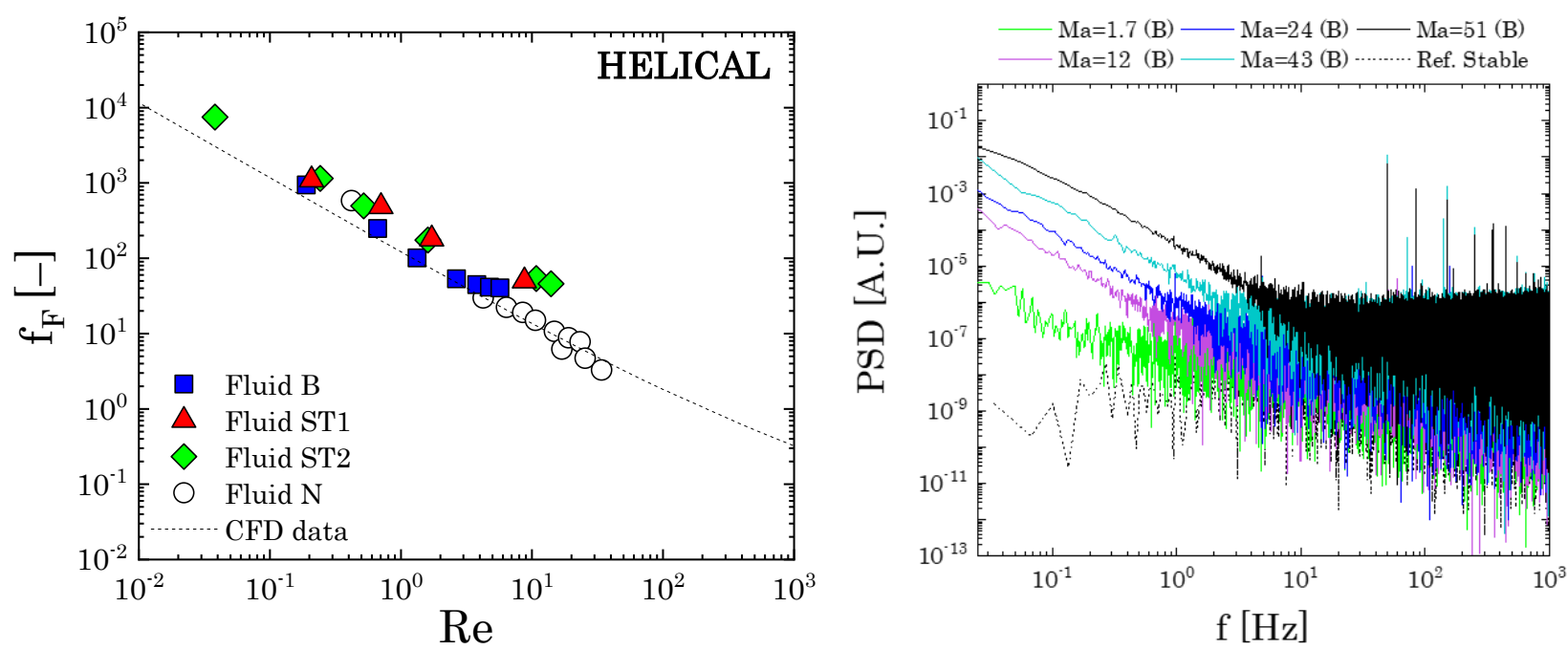

(a)
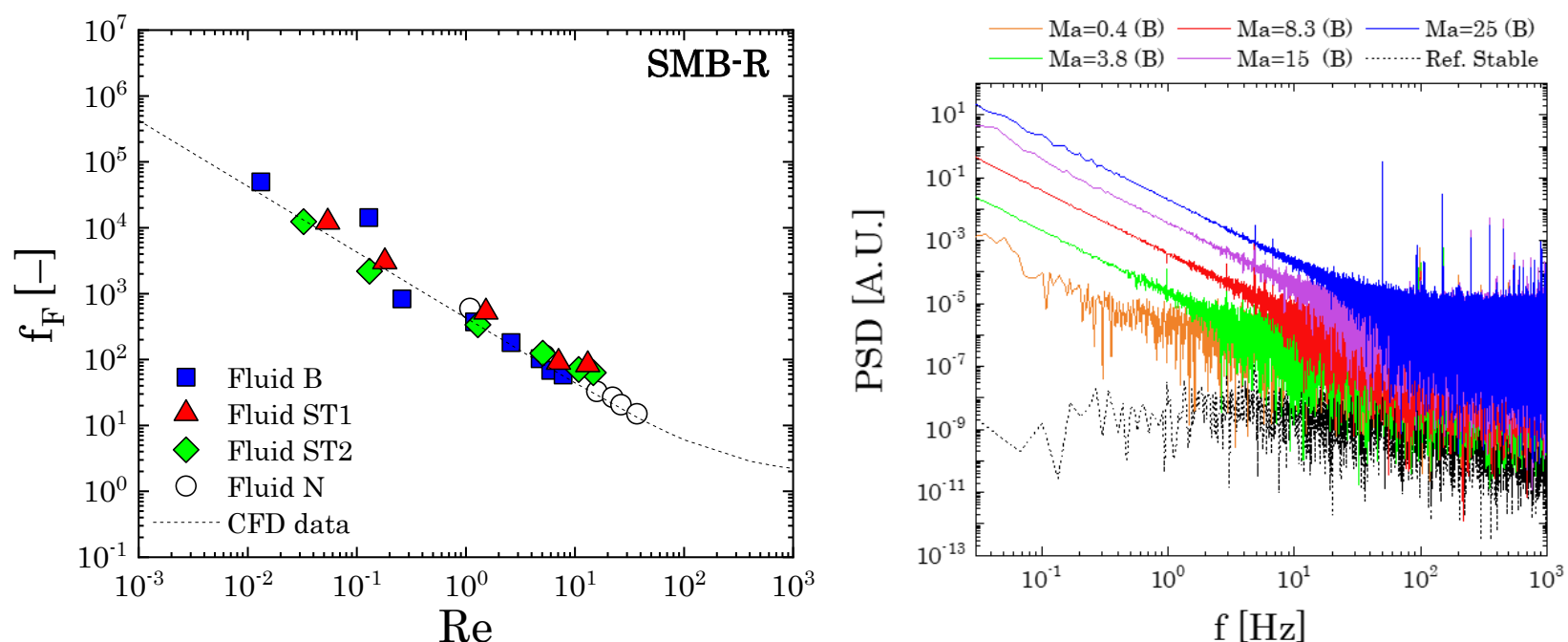

(b)

FIG. 15. (a) Friction factor vs. Re for all fluid tested (left) and sample PSD functions at increasing Ma for fluid B (the qualitative behaviour is the same for all fluid tested) (right) for the helical geometry; (b) Friction factor vs. Re for all fluid tested (left) and sample PSD functions at increasing Ma for fluid B (the qualitative behaviour is the same for all fluid tested) (right) for the SMB-R geometry.

and the SMB-R mixer. We captured instantaneous concentration profiles via Planar Laser Induced Fluorescence measurements at different flow rates, whilst pressure drop measurements allowed us to continuously monitor the flow resistance. Three different polymeric solutions were employed to investigate the effect of the interplay between the fluid rheological properties and the complex kinematics induced by the mixing elements on the mixing patterns. Specifically, a diluted solution of PAA in glycerol was used as Boger fluid to test the effects of pure elasticity, whilst two Xanthan gum solutions were used to investigate the combination of elasticity and shear-thinning behaviour.

Clear deviations from the Newtonian benchmark appeared as soon as the Deborah number exceeded unity, even though, in both geometries different transitions were observed as the mean flow rate was increased. In the case of the helical mixer, two qualitative behaviours can be distinguished for all fluids: (i) one at intermediate De, where the initial streams are split in thinner striations, as many as in the Newtonian case, yet showing anomalous shapes; (ii) the other at higher De, where the streams are simply split in two striations with irregular shapes, independently of the number of mixing elements employed. In this high De regime, a time-dependent behaviour, characterised by local fluctuations of the concentration patterns, can also be observed. The striations preserve their original shapes, yet showing irregular vibration of their boundaries. These local oscillations have a significant chaotic character, identifiable from a characteristic power law decay of the corresponding PSD signals, which was not affected by the operating conditions. The onset of the time-dependent behaviour, which can be identified using the viscoelastic Mach number, varies with the fluid properties: for the purely elastic fluid, oscillations can be observed at $\mathrm{Ma} \approx 1$, whilst they are delayed to $\mathrm{Ma} \approx 5$ in the case of the mildly shear-thinning fluid and completely suppressed for the highly shear-thinning fluid. These results suggest that, if the solid-like behaviour of 
the fluid is significant (i.e. De $>1$ ), local flow disturbances can originate in the mixing area, as a result of the interplay between the elastic stresses and local extensional components of the flow induced by the mixing elements, which then propagate downstream. Nonetheless, the scale of the perturbation remains small compared to the whole pipe cross-section and, even if the local oscillations present the characteristics associated to elastic turbulence, the effects at the large scale are negligible. Hence, in the case of the helical geometry, the onset of unsteady instabilities does not influence the mixing efficiency of the process, which is, instead, controlled by the efficiency of the splitting/stretching mechanism of the liquid streams, similarly to the Newtonian case.

For the SMB-R mixer, the transitions to different regimes are more complex. In the case of the Boger fluid, the transitions observed are similar to the helical geometry, with the addition of a different regime appearing at $\mathrm{Ma}>10$, characterised by more elongated striations that strongly oscillate in time. The qualitative behaviour is maintained with an increase in the number of mixing elements, even though the unsteady transitions appear at lower Ma. This suggests the occurrence of a first flow instability, generated downstream of the mixing element at lower Ma, followed by the formation of upstream vortexes, which can interact and destabilise, generating strong fluctuations of the concentration. On the other hand, for the shear-thinning solutions, strong oscillations, which become progressively more chaotic with an increase of the mean flow rate, are observed also at $\mathrm{Ma}<1$, thus suggesting the onset of dead zones instabilities ${ }^{37}$. The addition of mixing elements appears to simply enhance the chaotic character of the fluctuations. Differently from the helical geometry, the onset of the instabilities does not have the same detrimental effect on the mixing efficiency, although formation of large dead zones in the mixing area can critically impact the process, creating large pools of unmixed liquids. In addition, in all conditions presenting strong temporal variations, the performances of the mixer are severely uneven in time.

In both geometries, the enhanced solid-like behaviour that the viscoelastic solutions assume with an increase in the mean flow rate strongly affects the stretching of the fluid elements and therefore the final shape of the mixing pattern. This effect greatly disturbs the robustness and reproducibility of the concentration profiles that are typical of static mixers in laminar conditions and resulted in a deterioration of the mixing quality for the systems investigated here. The results highlight the necessity to further investigate the impact of both elasticity and extensional properties of the fluid on the operation of continuous flow mixing technologies.

\section{SUPPLEMENTARY INFORMATION}

Supplementary information are available in a separate file containing: (i) design details of the two mixers employed; (ii)calibration curve for PLIF experiments; (iii) estimate of the overlapping concentration of the polymer solutions; (iv) CFD simulation for the Newtonian case; (v) additional maps for SMB-R geometry.

\section{ACKNOWLEDGMENTS}

We wish to acknowledge Dr. Kassandra Makri and the Chemical Engineering Workshop at UCL for the help in setting up the experimental apparatus and GlaxoSmithKline Consumer Healthcare and the EPSRC (Formulation grant EP/N024915/1) for the financial support given to this project.

\section{DATA AVAILABILITY}

The data that support the findings of this study are available from the corresponding authors upon reasonable request.

\section{REFERENCES}

${ }^{1}$ P. E. Dimotakis, “Turbulent Mixing," Annual Review of Fluid Mechanics 37, 329-356 (2005).

${ }^{2} \mathrm{~J}$. M. Ottino, The kinematics of mixing : stretching , chaos , and transport (Cambridge University Press, Cambridge, 1989)

${ }^{3}$ P. D. Swanson and J. M. Ottino, "A comparative computational and experimental study of chaotic mixing of viscous fluids," Journal of Fluid Mechanics 213, 229-249 (1990).

${ }^{4} \mathrm{~S}$. W. Jones, "The enhancement of mixing by chaotic advection," Physics of Fluids A: Fluid Dynamics 3, 1081-1086 (1991).

${ }^{5}$ P. E. Arratia and F. J. Muzzio, "Planar Laser-Induced Fluorescence Method for Analysis of Mixing in Laminar Flows," Ind. Eng. Chem. Res 43, 6557 6568 (2004).

${ }^{6}$ G. Fountain, D. Khakhar, I. Mezic, and J. M. Ottino, "Chaotic mixing in a bounded three-dimensional flow," Journal of Fluid Mechanics 417, 265 301 (2000).

${ }^{7}$ E. L. Paul, V. A. Atiemo-Obeng, and S. M. Kresta, Handbook of Industrial mixing - Science and practice (John Wiley \& Sons, Inc, Hoboken, New Jersey, 2004).

${ }^{8}$ R. K. Thakur, C. Vial, K. D. P. Nigam, E. B. Nauman, and G. Djelveh, "Static mixers in the process industries - a review," Chemical Engineering Research and Design 81, 787 - 826 (2003).

${ }^{9}$ D. M. Hobbs and F. J. Muzzio, "The Kenics static mixer: A threedimensional chaotic flow," Chemical Engineering Journal 67, 153-166 (1997).

${ }^{10} \mathrm{O}$. Wunsch and G. Bohme, "Numerical simulation of 3D viscous fluid flow and convective mixing in a static mixer," Archive of Applied Mechanics 70, $91-102(2000)$

${ }^{11}$ O. Galaktionov, P. Anderson, G. Peters, and H. Meijer, "Morphology development in kenics static mixers (Application of the extended mapping method)," Canadian Journal of Chemical Engineering 80, 604-613 (2002).

${ }^{12}$ E. S. Szalai and F. J. Muzzio, "Fundamental Approach to the Design and Optimization of Static Mixers," AIChE Journal 49, 2687-2699 (2003).

${ }^{13}$ J. M. Zalc, E. S. Szalai, and F. J. Muzzio, "Mixing dynamics in the SMX static mixer as a function of injection location and flow ratio," Polymer Engineering \& Science 43, 875-890 (2003).

${ }^{14}$ R. K. Rahmani, A. Ayasoufi, and T. G. Keith, "A Numerical Study of the Global Performance of Two Static Mixers," Journal of Fluids Engineering 129, 338 - 349 (2007)

${ }^{15}$ S. Y. Jung, K. H. Ahn, T. G. Kang, G. T. Park, and S. U. Kim, "Chaotic Mixing in a Barrier-Embedded Partitioned Pipe Mixer,” AIChE Journal 64, 717-729 (2018)

${ }^{16}$ J. M. Ottino, F. J. Muzzio, M. Tjahjadi, J. G. Franjione, S. C. Jana, and H. A. Kusch, "Chaos, Symmetry, and Self-Similarity : Exploiting Order and Disorder in Mixing Processes," Science 257, 754 -760 (1992).

${ }^{17}$ W. E. Ranz, "Applications of a Stretch Model to Mixing , Diffusion , and Reaction in Laminar and Turbulent Flows," AIChE Journal 25, 41-47 (1979).

${ }^{18}$ M. Speetjens, G. Metcalfe, and M. Rudman, "Topological mixing study of non-Newtonian duct flows," Physics of Fluids 18, 1 - 11 (2006). 
${ }^{19}$ V. V. Meleshko, O. S. Galaktionov, G. W. M. Peters, and H. E. H. Meijer, "Three-dimensional mixing in Stokes flow: The partitioned pipe mixer problem revisited," European Journal of Mechanics, B/Fluids 18, 783-792 (1999).

${ }^{20}$ T. Avalosse and M. J. Crochet, "Finite-Element Simulation of Mixing : 2. Three-Dimensional Flow Through a Kenics Mixer," AIChE Journal 43, 588 - 597 (1997).

${ }^{21}$ S. Liu, A. N. Hrymak, and P. E. Wood, "Laminar mixing of shear thinning fluids in a SMX static mixer," Chemical Engineering Science 61, 17531759 (2006).

${ }^{22}$ V. Kumar, V. Shirke, and K. D. Nigam, "Performance of Kenics static mixer over a wide range of Reynolds number," Chemical Engineering Journal 139, 284-295 (2008).

${ }^{23}$ V. Lim, A. M. Hobby, M. J. McCarthy, and K. L. McCarthy, "Laminar mixing of miscible fluids in a SMX mixer evaluated by magnetic resonance imaging (MRI)," Chemical Engineering Science 137, 1024-1033 (2015).

${ }^{24} \mathrm{G}$. Böhme and O. Wünsch, "Analysis of shear-thinning fluid flow in intermeshing twin-screw extruders," Archive of Applied Mechanics 67, 167178 (1997).

${ }^{25}$ R. K. Rahmani, T. G. Keith, and A. Ayasoufi, "Numerical Simulation and Mixing Study of Pseudoplastic Fluids in an Industrial Helical Static Mixer," Journal of Fluids Engineering 128, 467-480 (2006).

${ }^{26}$ R. B. Bird, R. C. Armostrong, and O. Hassanger, Dynamics of polymer liquids - Fluid mechanics, 2nd ed. (1987).

${ }^{27}$ R. G. Larson, E. S. G. Shaqfeh, and S. J. Muller, “A purely elastic instability in Taylor-Couette flow," Journal of Fluid Mechanics 218, 573-800 (1990).

${ }^{28}$ J. A. Byars, A. Oztekin, R. A. Brown, and G. H. McKinley, "Spiral instabilities in the flow of highly elastic fluids between rotating parallel disks,' Journal of Fluid Mechanics 271, 173-218 (1994).

${ }^{29}$ B. M. Baumert and S. J. Muller, "Flow visualization of the elastic TaylorCouette instability in Boger fluids," Rheologica Acta 34, 147-159 (1995).

${ }^{30}$ E. S. G. Shaqfeh, "Purely elastic instabilities in viscometric flow," Annual Review of Fluid Mechanics 28, 129 - 185 (1996).

${ }^{31}$ K. Chiba, T. Sakatani, and K. Nakamura, "Anomalous flow patterns in viscoelastic entry flow through a planar contraction," Journal of NonNewtonian Fluid Mechanics 36, 193-203 (1990).

${ }^{32}$ L. Skartsis, B. Khomami, and J. L. Kardos, "Polymeric flow through fibrous media," Journal of Rheology 36, 589 - 620 (1992)

${ }^{33}$ D. L. Davidson, W. Graessley, and W. Showalter, "Velocity and stress fields of polymeric liquids flowing in a periodically constricted channel - Part 2 . Observations of non-Newtonian behavior," Journal of Non-Newtonian Fluid Mechanics 49, 345-375 (1993).

${ }^{34}$ I. M. Dris and E. S. G. Shaqfeh, "Experimental and theoretical observations of elastic instabilities in eccentric cylinder flows : local versus globa instability," Journal of Non-Newtonian Fluid Mechanics 80, 1-58 (1998).

${ }^{35}$ K. Arora, R. Sureshkumar, and B. Khomami, "Experimental investigation of purely elastic instabilities in periodic flows," Journal of Non-Newtonian Fluid Mechanics 108, 209-226 (2002).

${ }^{36}$ G. R. Moss and J. P. Rothstein, "Flow of wormlike micelle solutions through a periodic array of cylinders," Journal of Non-Newtonian Fluid Mechanics 165, 1-13 (2010).

${ }^{37}$ D. Kawale, E. Marques, P. L. Zitha, M. T. Kreutzer, W. R. Rossen, and P. E. Boukany, "Elastic instabilities during the flow of hydrolyzed polyacrylamide solution in porous media: effect of pore-shape and salt," Soft Matter 13, 765-775 (2017).

${ }^{38}$ R. Larson, "Instabilities in viscoelastic flows," Rheologica Acta 31, 213 263 (1992)

${ }^{39}$ A. Oztekin, R. A. Brown, and G. H. Mckinley, "Quantitative prediction of the viscoelastic instability in cone-and-plate flow of a Boger fluid using a multi-mode Giesekus model,' Journal of Non-Newtonian Fluid Mechanics 54, 351-377 (1994).

${ }^{40}$ G. H. Mckinley, P. Pakdel, and A. Oztekin, "Rheological and geometric scaling of purely elastic flow instabilities," Journal of Non-Newtonian Fluid Mechanics 67, 19-47 (1996).

${ }^{41}$ P. Pakdel and G. H. Mckinley, "Elastic Instability and Curved Streamlines," Physical Review Letters 77, 2459-2462 (1996).

${ }^{42}$ T. C. Niederkorn and J. M. Ottino, "Mixing of a viscoelastic fluid in a timeperiodic flow," Journal of Fluid Mechanics 256, 243 - 268 (1993).

${ }^{43}$ P. E. Arratia, G. A. Voth, and J. P. Gollub, "Stretching and mixing of nonNewtonian fluids in time-periodic flows," Physics of Fluids 17, 053102-(1-
10) (2005).

${ }^{44}$ A. Groisman and V. Steinberg, "Efficient mixing at low Reynolds numbers using polymer additives," Nature 410, 905-908 (2001).

${ }^{45}$ M. Grilli, A. Vazquez-Quesada, and M. Ellero, "Transition to Turbulence and Mixing in a Viscoelastic Fluid Flowing Inside a Channel with a Periodic Array of Cylindrical Obstacles," Physical Review Letters 110, 174501 (15) (2013)

${ }^{46}$ J. R. Stokes and D. V. Boger, "Mixing of viscous polymer liquids," Physics of Fluids 12, 1411 - 1416 (2000).

${ }^{47}$ J. R. Stokes, L. J. W. Graham, N. J. Lawson, and D. V. Boger, "Swirling flow of viscoelastic fluids. Part 2. Elastic effects," Journal of Fluid Mechanics 429, 117-153 (2001)

${ }^{48}$ L. Pan, A. Morozov, C. Wagner, and P. E. Arratia, "Nonlinear Elastic Instability in Channel Flows at Low Reynolds Numbers," Physical Review Letters 110, 174502-(1-5) (2013).

${ }^{49}$ J. Ramsay, M. J. H. Simmons, A. Ingram, and E. H. Stitt, "Mixing performance of viscoelastic fluids in a Kenics KM in-line static mixer," Chemical Engineering Research and Design 115, 310-324 (2016).

${ }^{50}$ D. M. Hobbs and F. J. Muzzio, "Reynolds number effects on laminar mixing in the Kenics static mixer," Chemical Engineering Journal 70, 93-104 (1998).

${ }^{51}$ O. Byrde and M. L. Sawley, "Optimization of a Kenics static mixer for non-creeping flow conditions," Chemical Engineering Journal 72, 163-169 (1999).

${ }^{52}$ H. E. Meijer, M. K. Singh, and P. D. Anderson, "On the performance of static mixers: A quantitative comparison,” Progress in Polymer Science 37 1333-1349 (2012).

${ }^{53}$ I. Houcine, H. Vivier, E. Plasari, R. David, and J. Villermaux, "Planar laser induced fluorescence technique for measurements of concentration fields in continuous stirred tank reactors," Experiments in Fluids 22, 95-102 (1996).

${ }^{54}$ N. Shah and D. D. Kale, "Pressure drop for laminar flow of viscoeleastic fluids in static mixers," Chemical Engineering Science 47, 2097-2100 (1992).

${ }^{55}$ J. B. Segur and H. E. Oderstar, "Viscosity of Glycerol and Its Aqueous Solutions," Industrial and Engineering Chemistry 43, 2117-2120 (1951).

${ }^{56}$ L. M. Quinzani, G. H. McKinley, R. A. Brown, and R. C. Armstrong, "Modeling the rheology of polyisobutylene solutions," Journal of Rheology 34, 705-748 (1990).

${ }^{57}$ D. F. James, "Boger Fluids," Annual Review of Fluid Mechanics 41, 129 142 (2009).

${ }^{58}$ J. R. Stokes, L. J. Graham, N. J. Lawson, and D. V. Boger, "Swirling flow of viscoelastic fluids. Part 1. Interaction between inertia and elasticity," Journal of Fluid Mechanics 429, 67-115 (2001).

${ }^{59}$ C. W. Macosko, Rheology: Principles, Measurements and Applications (Wiley-VCH, Inc, 1994).

${ }^{60}$ R. Poole, "The Deborah and Weissenberg numbers," The British Society of Rheology, Rheology Bulletin 53, 32-39 (2012).

${ }^{61}$ S. Kenney, K. Poper, G. Chapagain, and G. F. Christopher, "Large Deborah number flows around confined microfluidic cylinders," Rheologica Acta 52, 485-497 (2013).

${ }^{62}$ X. Shi and G. F. Christopher, "Growth of viscoelastic instabilities around linear cylinder arrays," Physics of Fluids 28 (2016), 10.1063/1.4968221.

${ }^{63} \mathrm{~J}$. Yoo and D. Joseph, "Hyperbolicity and change of type in the flow of viscoelastic fluids through channels," Journal of Non-Newtonian Fluid Mechanics 19, 19-41 (1985).

${ }^{64}$ D. M. Hobbs, P. D. Swanson, and F. J. Muzzio, "Numerical characterization of low Reynolds number flow in the Kenics static mixer," Chemical Engineering Science 53, 1565-1584 (1998).

${ }^{65} \mathrm{M}$. Regner, K. Östergren, and C. Trägårdh, "Effects of geometry and flow rate on secondary flow and the mixing process in static mixers-a numerical study," Chemical Engineering Science 61, 6133-6141 (2006).

${ }^{66}$ M. Milas, M. Rinaudo, M. Knipper, and J. L. Schuppiser, "Flow and Viscoelastic Properties of Xanthan Gum Solutions," Macromolecules 23 2506-2511 (1990).

${ }^{67}$ A. B. Rodd, D. E. Dunstan, and D. V. Boger, "Characterization of xanthan gum solutions using dynamic light scattering and rheology," Carbohydrate Polymers 42, 159-174 (2000).

${ }^{68}$ S. J. Haward and G. H. McKinley, "Instabilities in stagnation point flows of polymer solutions,” Physics of Fluids 25 (2013), 10.1063/1.4818151. 
${ }^{69}$ B. Khomami and L. D. Moreno, "Stability of viscoelastic flow around periodic arrays of cylinders," Rheologica Acta 36, 367-383 (1997).

${ }^{70}$ A. Groisman and V. Steinberg, "Elastic turbulence in curvilinear flows of polymer solutions," New Journal of Physics 6 (2004), 10.1088/13672630/6/1/029.

${ }^{71}$ T. Burghelea, E. Segre, I. Bar-Joseph, A. Groisman, and V. Steinberg, "Chaotic flow and efficient mixing in a microchannel with a polymer solution," Physical Review E - Statistical Physics, Plasmas, Fluids, and Related Interdisciplinary Topics 69, 8 (2004).
${ }^{72}$ F. C. Li, H. Kinoshita, X. B. Li, M. Oishi, T. Fujii, and M. Oshima, "Creation of very-low-Reynolds-number chaotic fluid motions in microchannels using viscoelastic surfactant solution," Experimental Thermal and Fluid Science 34, 20-27 (2010).

${ }^{73}$ Y. Zhao, A. Q. Shen, and S. J. Haward, "Flow of wormlike micellar solutions around confined microfluidic cylinders," Soft Matter 12, 8666-8681 (2016)

${ }^{74}$ B. Qin, P. F. Salipante, S. D. Hudson, and P. E. Arratia, "Upstream vortex and elastic wave in the viscoelastic flow around a confined cylinder," Journal of Fluid Mechanics 864, R2/1-11 (2019). 

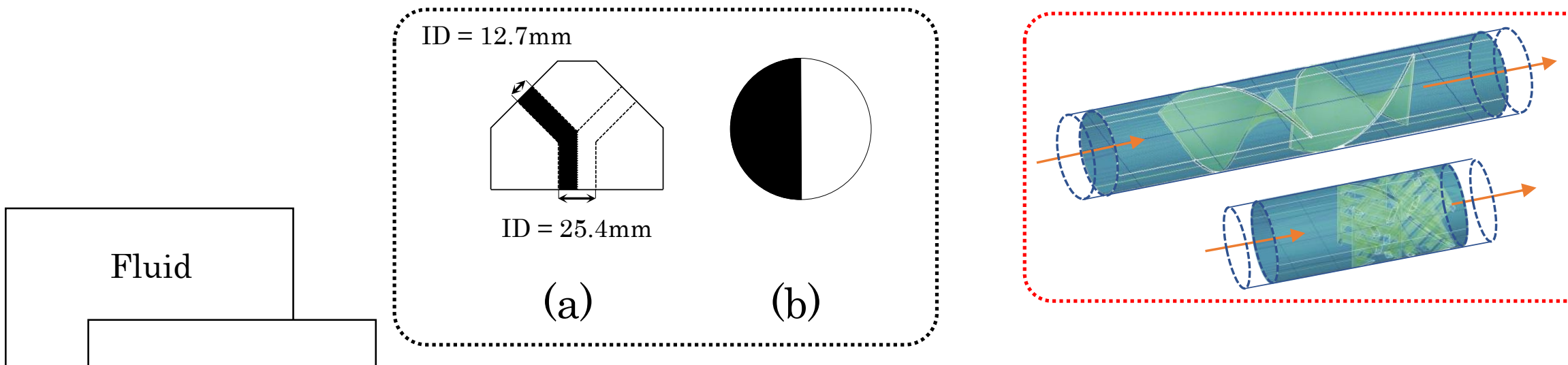

(c)

(d)

High-speed camera

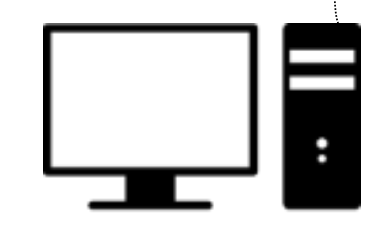

VISUALISATION SECTION

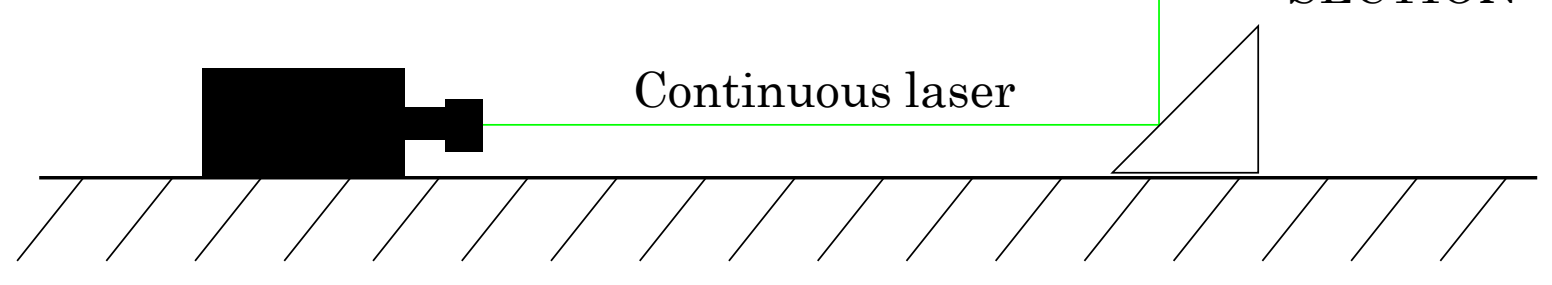




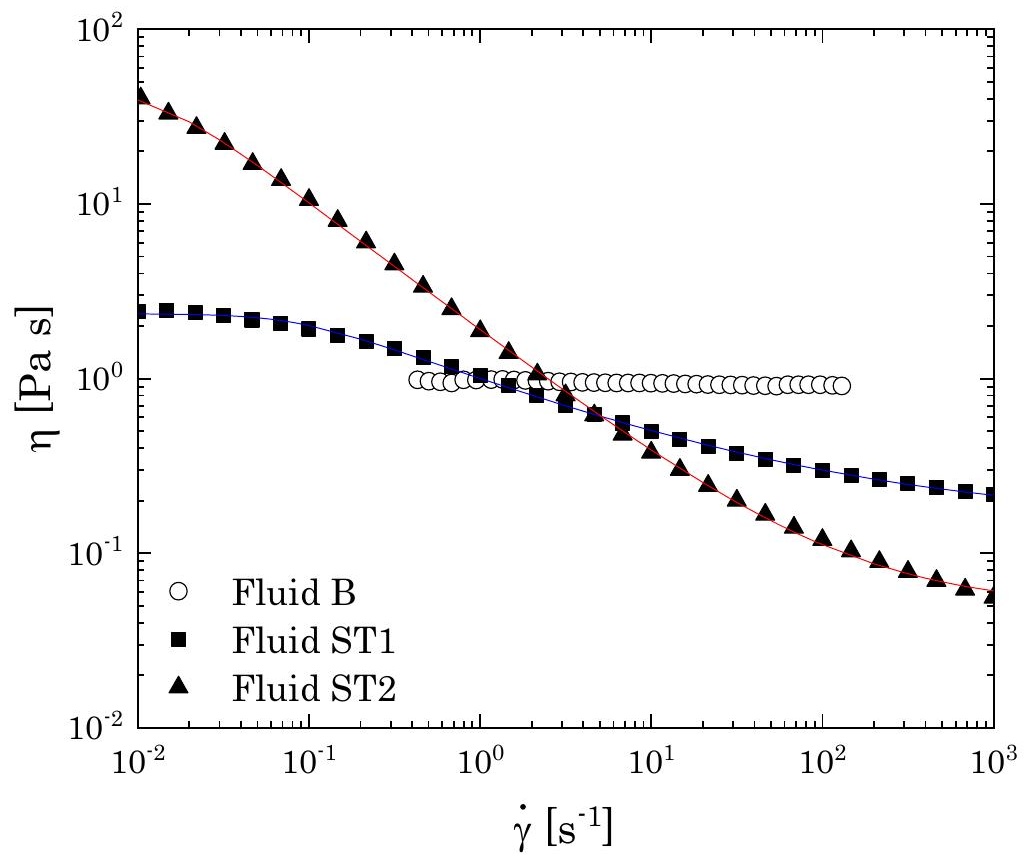



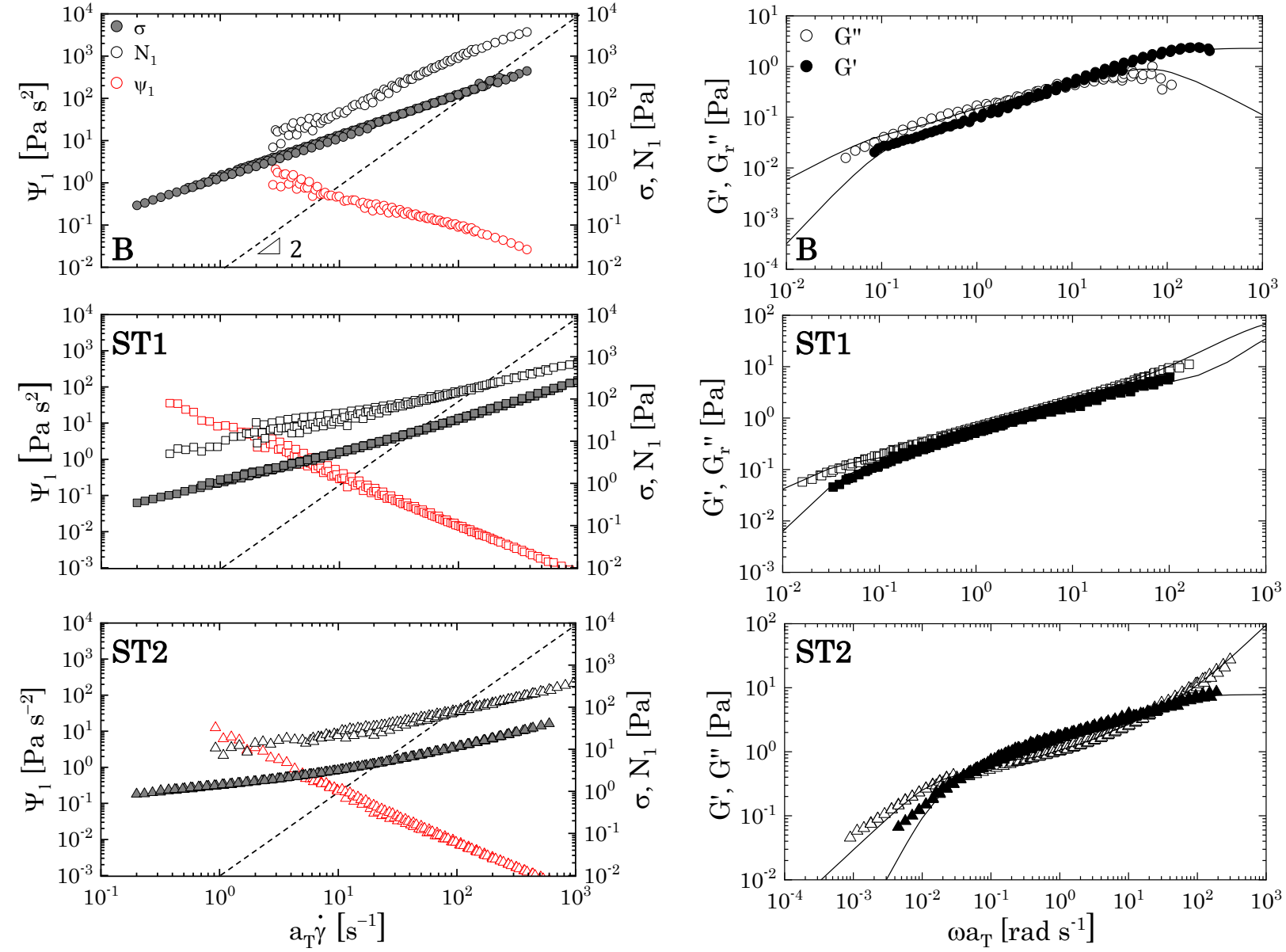

(a)
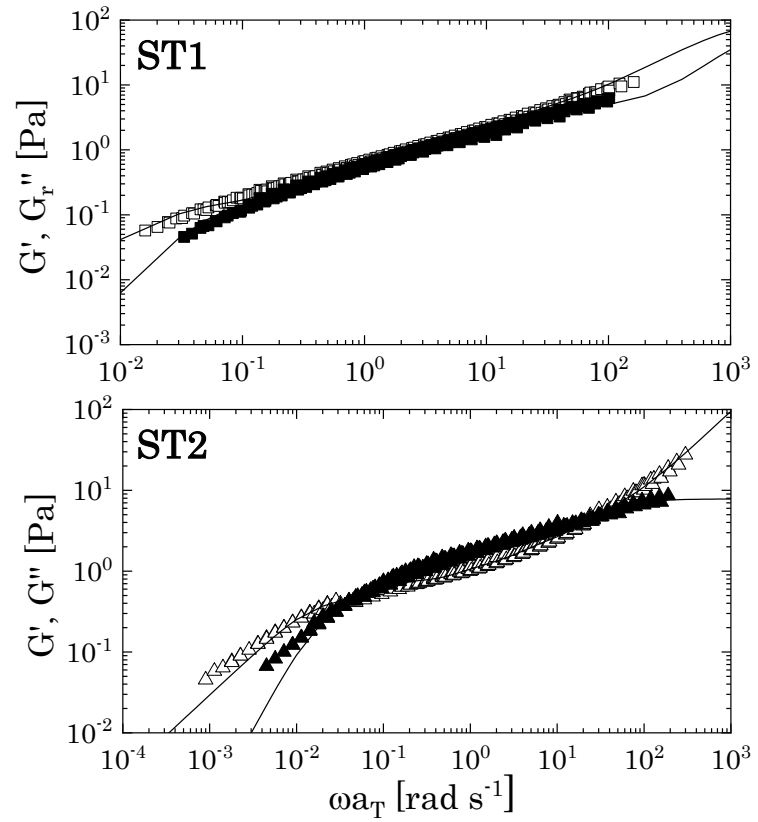

(b) 


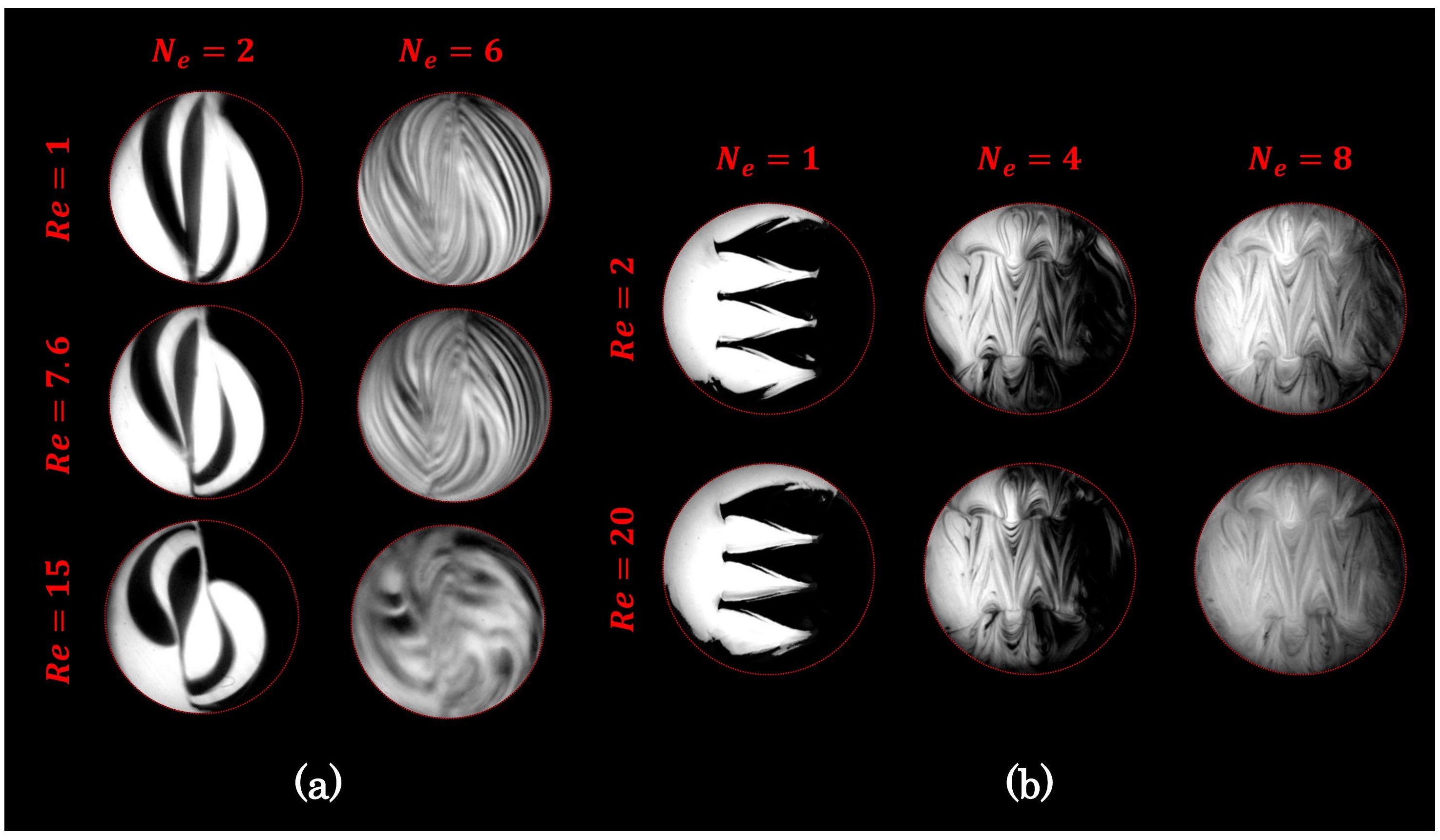



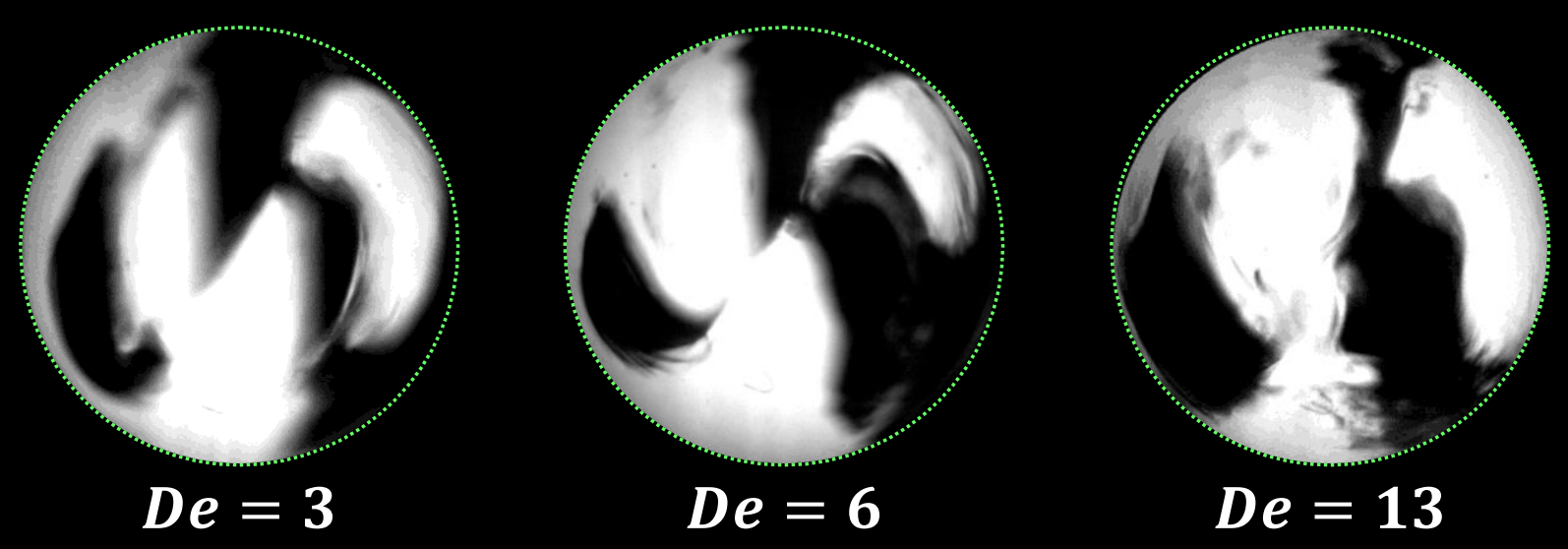

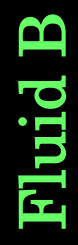
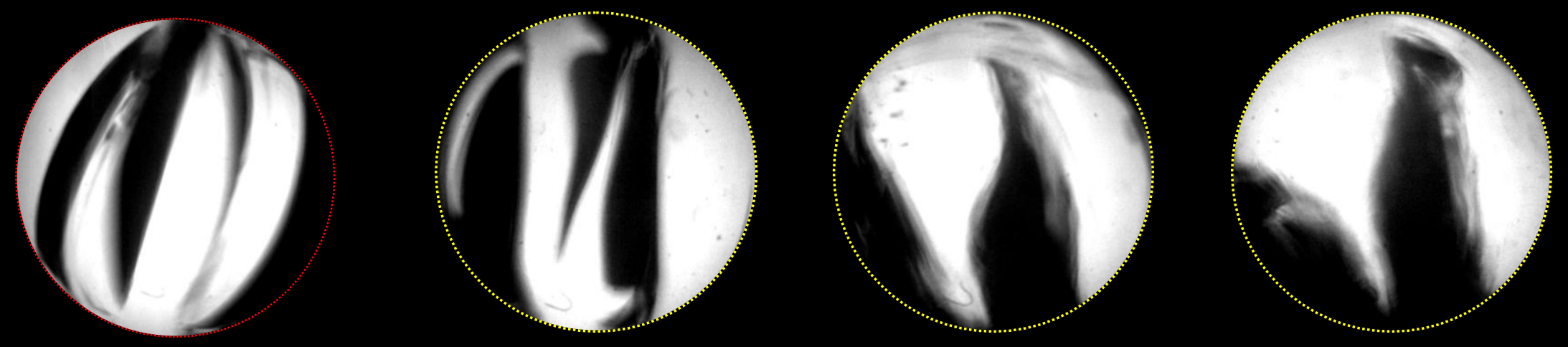

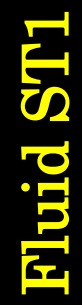

De $<1$

$D e=2.3$

$$
D e=12
$$

$$
D e=44
$$
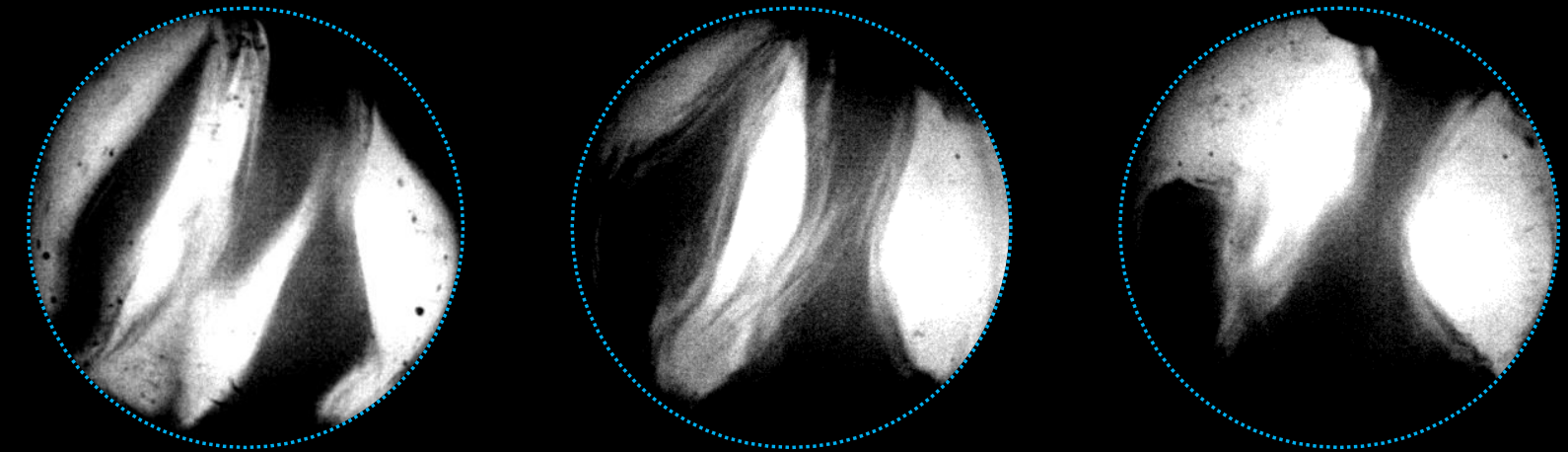

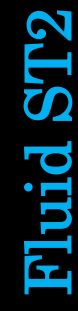

$D e=2.8$

$D e=25.8$

$D e=83.8$ 

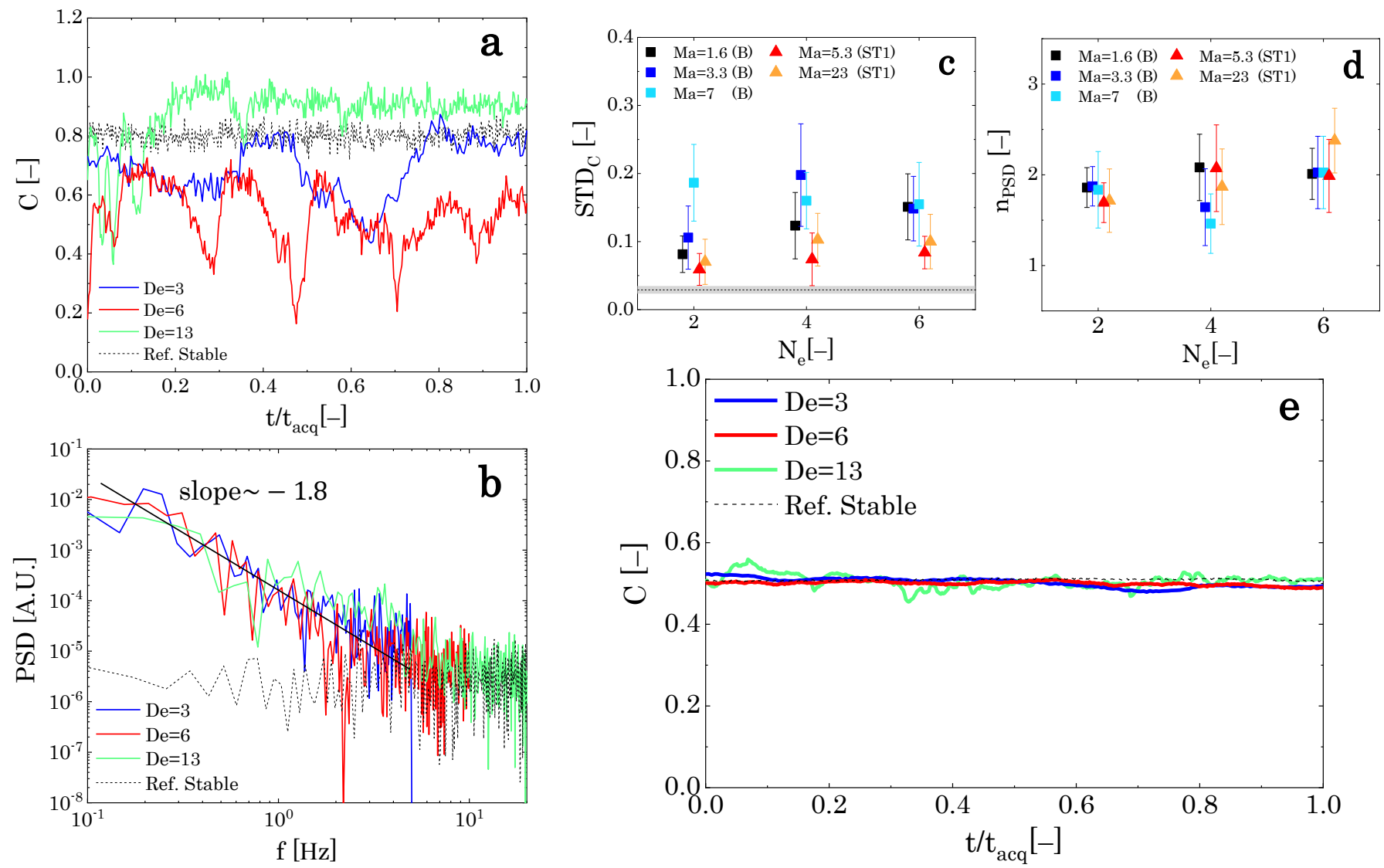


\section{$D e<1$}

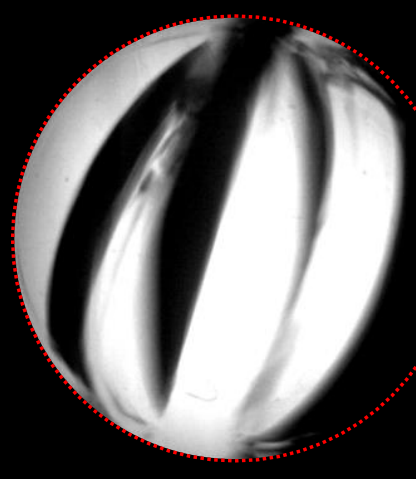

$$
1<D e<10
$$

\section{$D e>10$}
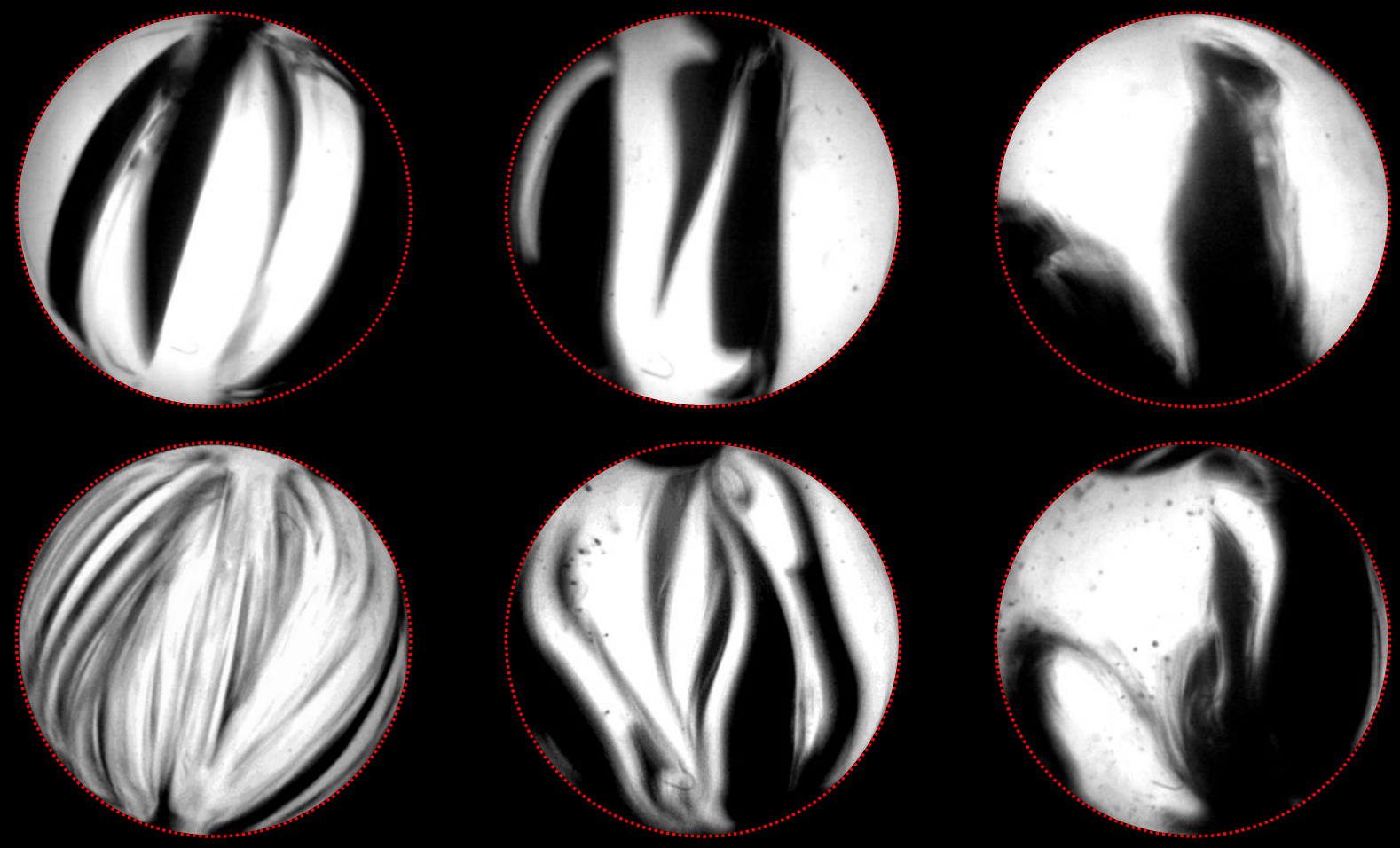

$\forall$
$n^{\prime}$

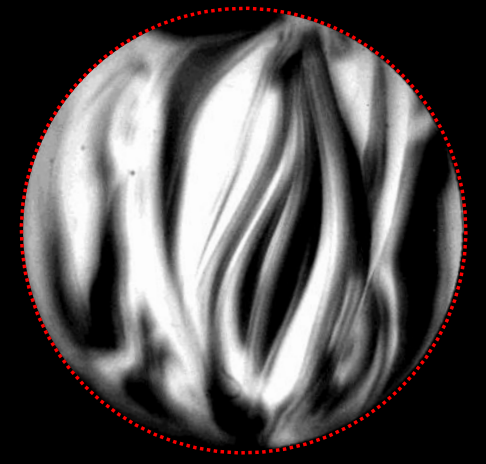

N

$z^{\prime \prime}$
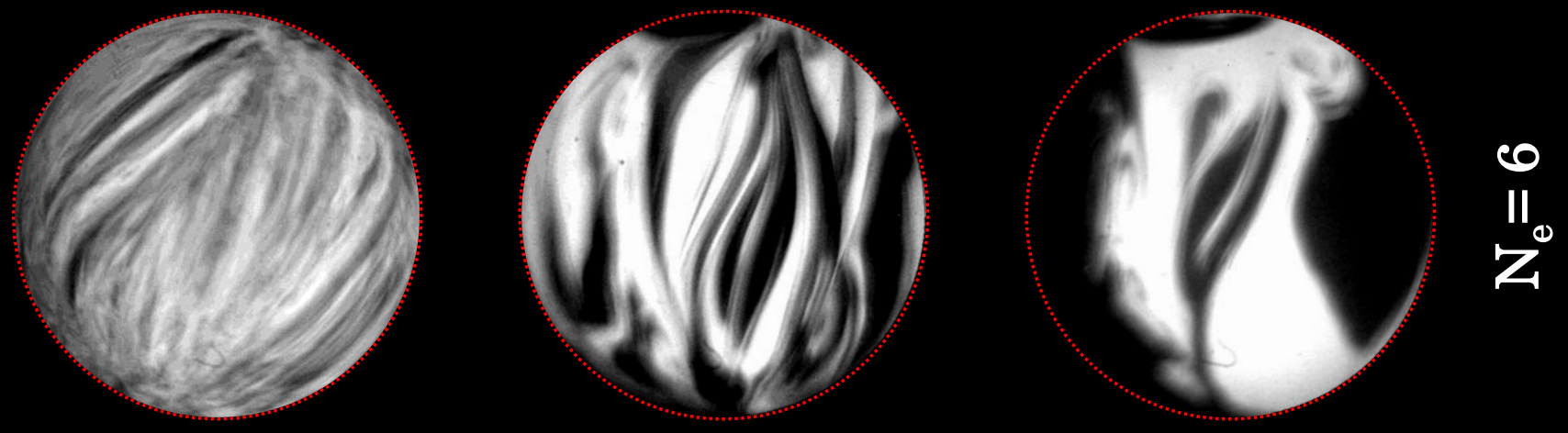


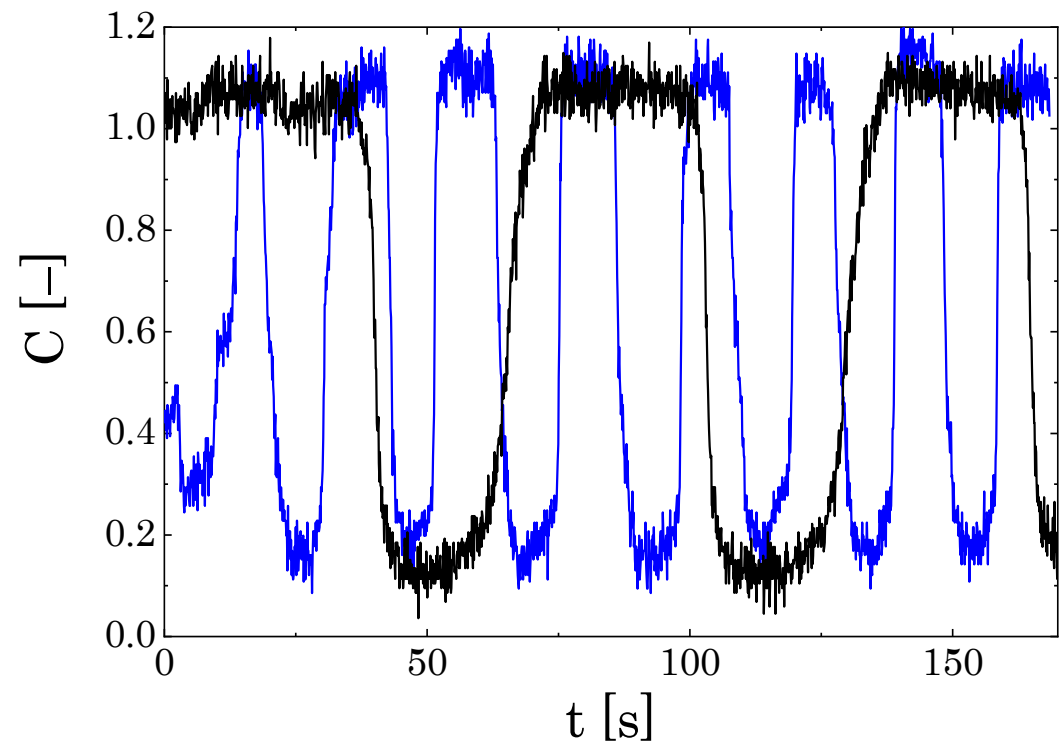

a)

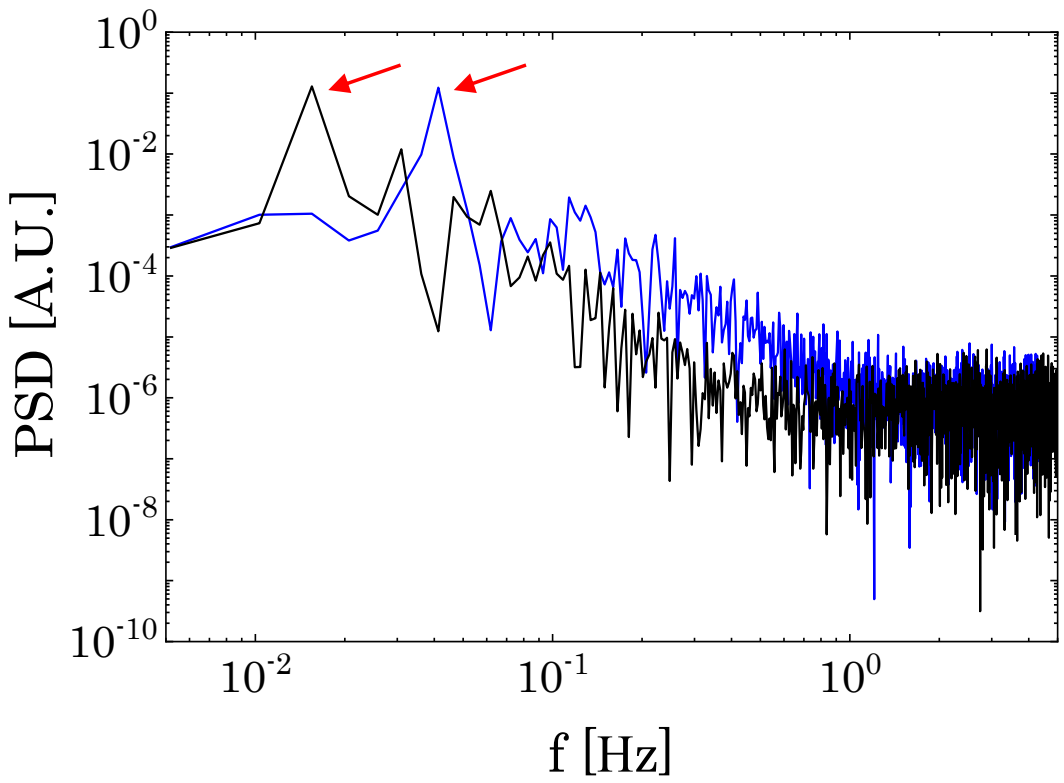




$$
N_{e}=1
$$

$$
\mathrm{N}_{\mathrm{e}}=4
$$

$N_{e}=8$
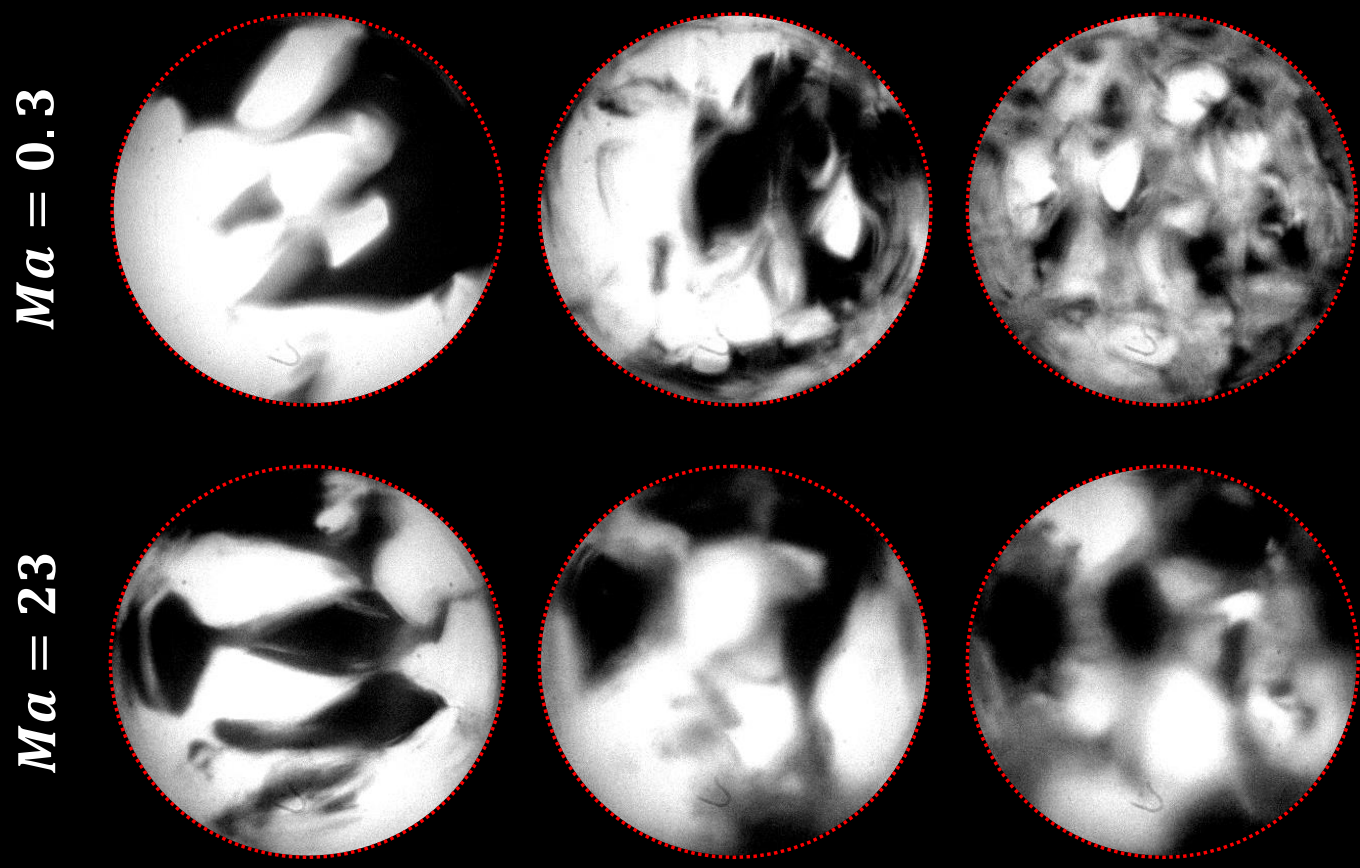


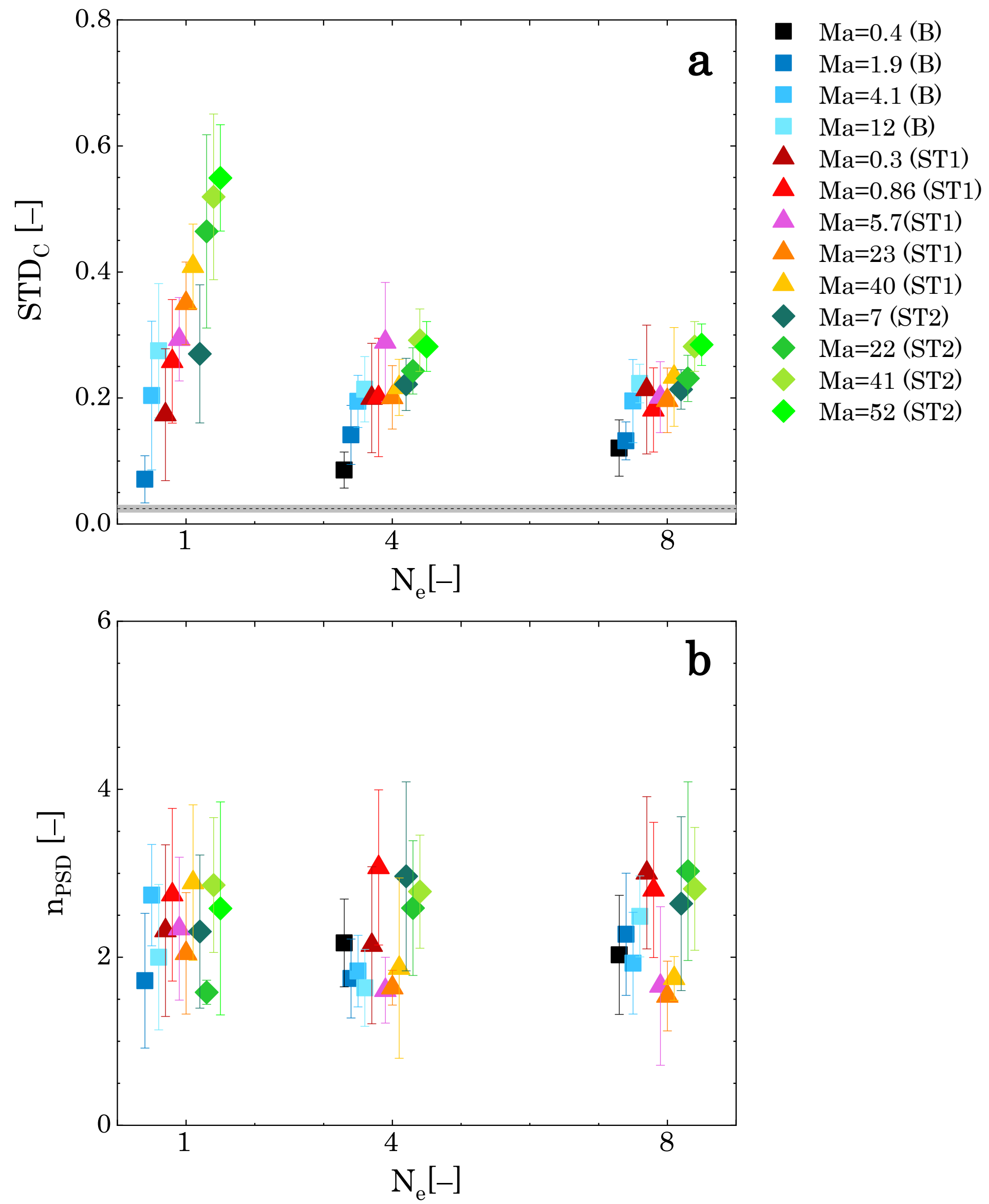



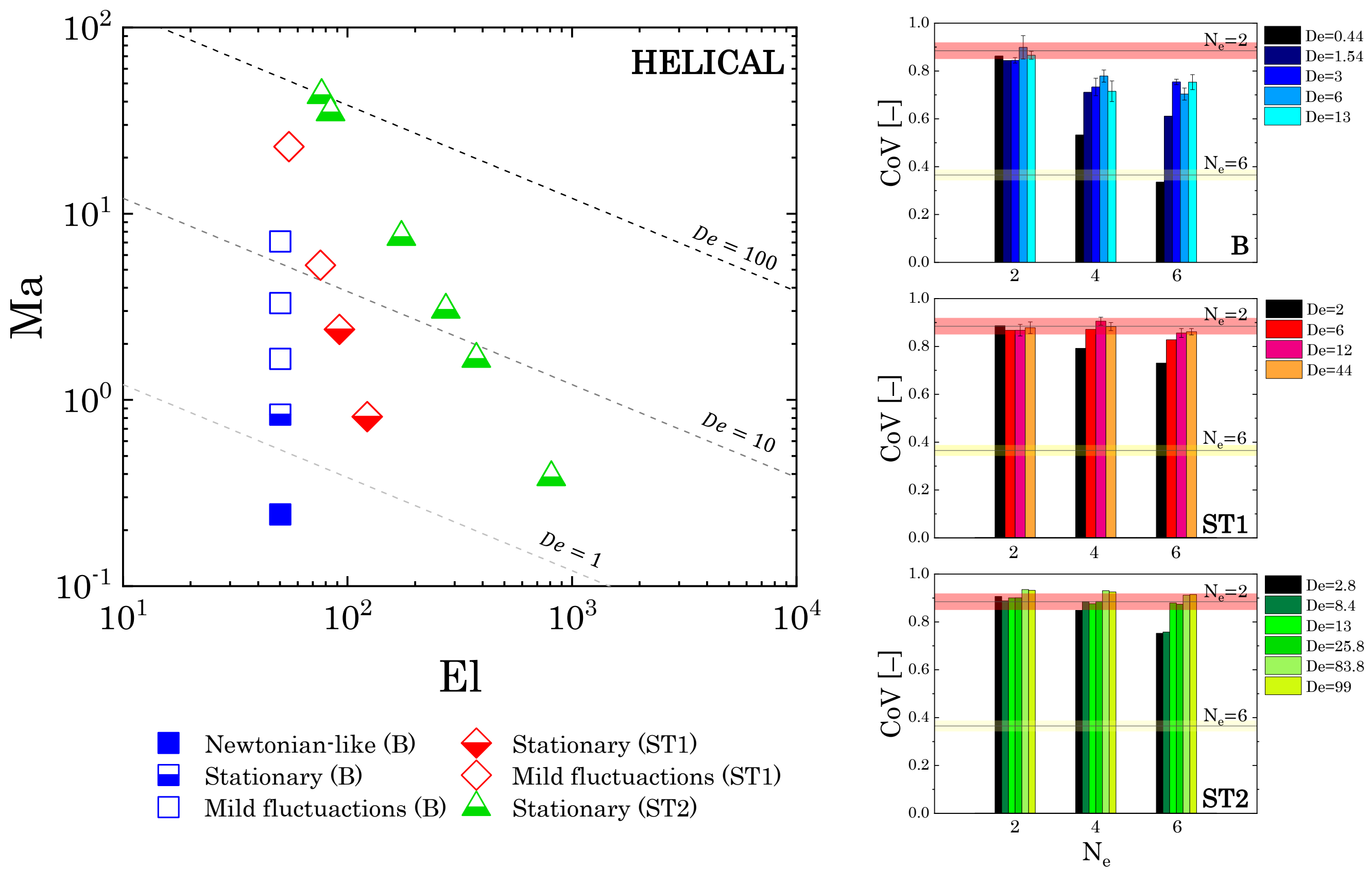

- Newtonian-like (B)

a Stationary (B)

$\square$ Mild fluctuactions (B)
Stationary (ST1)

Mild fluctuactions (ST1)

Stationary (ST2)

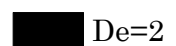

$\mathrm{De}=2$ $\mathrm{De}=12$ $\mathrm{De}=44$

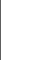

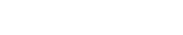


